\title{
MEASUREMENT AND ANALYSIS OF THE PHYSICAL AND CLIMATIC DISTRIBUTION ENVIRONMENT FOR AIR PACKAGE SHIPMENT
}

\author{
A Thesis \\ presented to \\ the Faculty of California Polytechnic State University, \\ San Luis Obispo
}

\author{
In Partial Fulfillment \\ of the Requirements for the Degree \\ Master of Science in Business and Technology
}

by

David Nathaniel Guadagnini

June 2017 
(C) 2017

David Nathaniel Guadagnini

\section{ALL RIGHTS RESERVED}




\section{COMMITTEE MEMBERSHIP}

TITLE: Measurement and Analysis of the Physical and Climatic Distribution Environment for Air Package Shipment

AUTHOR: David Nathaniel Guadagnini

DATE SUBMITTED: June 2017

COMMITTEE CHAIR: Jay Singh, Ph.D.

Professor of Industrial Technology and Packaging

COMMITTEE MEMBER: Koushik Saha, Ph.D.

Associate Professor of Industrial Technology and Packaging

COMMITTEE MEMBER: Ajay Kathuria, Ph.D.

Assistant Professor of Industrial Technology and Packaging 


\begin{abstract}
Measurement and Analysis of the Physical and Climatic Distribution

Environment for Air Package Shipment
\end{abstract}

David Nathaniel Guadagnini

The modern air parcel distribution industry has significantly grown to become one of the most commonly employed methods to quickly transport goods throughout the world. Although it comes with many benefits, including higher speed, greater reliability, and tighter security, the multimodal transport system within it can expose packages to a wide variety of climatic and physical distribution hazards. In a single route of transportation, packages could be included in different types of small delivery vans, large commercial semi trucks, cargo dollies, feeder aircraft, and high altitude commercial jetliners. The varying hazard level presented during distribution could directly weaken the packaging components and/ or cause product damage. For this reason, it is of utmost importance to properly account for them during package design.

Although there have been many past studies to quantify the hazards experienced in specific modes of transport, an over-arching profile of entire distribution route has not yet been developed. Furthermore, after a review of the current testing standards presented in the Code of Federal Regulations as outlined in 49 CFR Part 178, Subpart $\mathrm{M}$, it can be found that many of these currently used testing profiles are not truly representative of the conditions experienced in actual distribution. This study quantifies each hazard element experienced within the modern air parcel distribution environment and develops single testing profiles to accurately represent them.

In order to develop single testing profiles for each hazard element, instrumented test packages were sent to multiple domestic and international destinations. Throughout each of these distribution routes, data was collected on the hazard levels experienced. Afterwards, by identifying the amount of time a package spends within each mode of transport, correctly weighted testing profiles were developed. These newly developed profiles represent the minimum hazard level to be included in package performance testing that represents the normal conditions of the air transport environment. Although these composite testing profiles are developed, it is the responsibility of testing laboratories to integrate these updated profiles into their practice. 


\section{ACKNOWLEDGMENTS}

I would like to thank the following people for their help with my master's thesis.

- Dr. Jay Singh- California Polytechnic State University

- Dr. Koushik Saha- California Polytechnic State University

- Dr. Ajay Kathuria- California Polytechnic State University

- Raymond Kisch- California Polytechnic State University

- Patrick Blizinski- Lansmont Corporation

- Michael Givens- Federal Aviation Administration

- Sanjit Singh- IntuiShip

- Evan Cernokus- Apple Inc.

- Francesca Delle Cese- Apple Inc.

- John Guadagnini- Life’s Innovations, LLC

- Kevin Guadagnini- Life's Innovations, LLC 


\section{TABLE OF CONTENTS}

Page

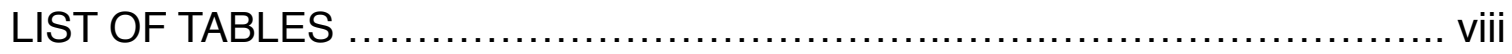

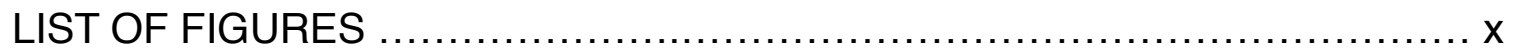

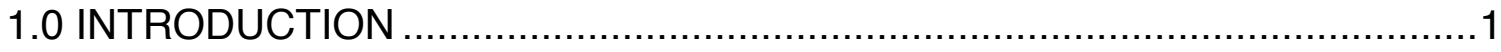

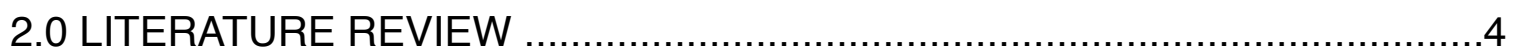

2.1 Air Package Distribution System ............................................... 4

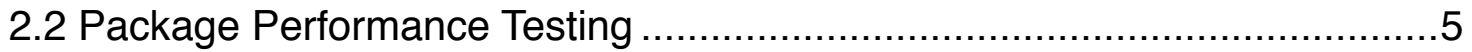

2.3 Climatic Distribution Hazards .......................................................

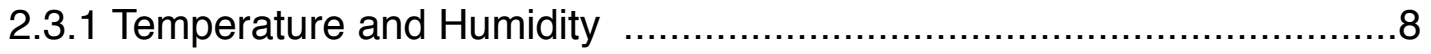

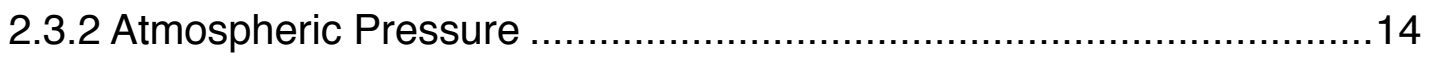

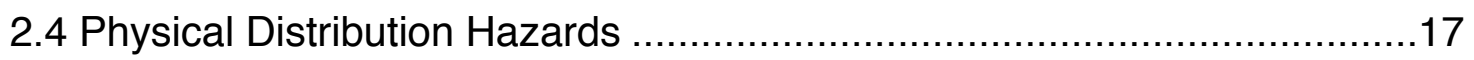

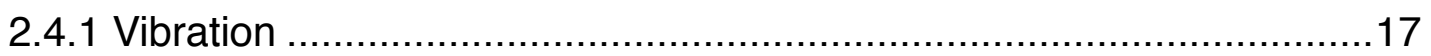

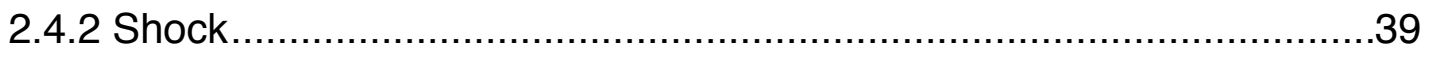

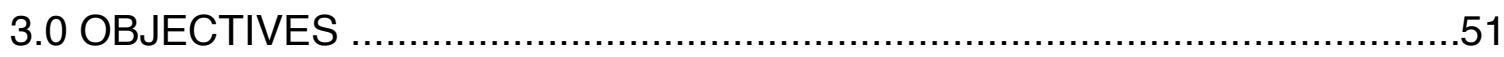

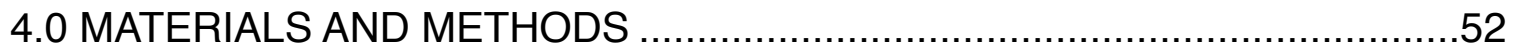

4.1 Data Recording Test Package Construction ..................................... 52

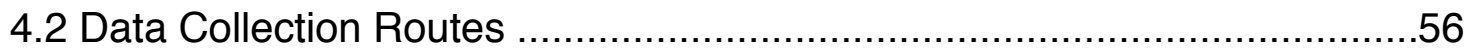

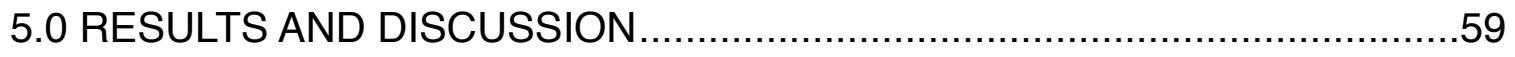


5.1 Climatic Hazard Findings

5.1.1 Suggested Climatic Hazard Testing Updates 63

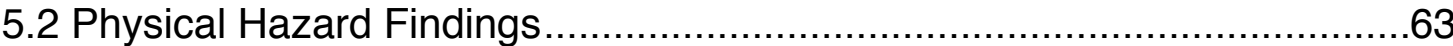

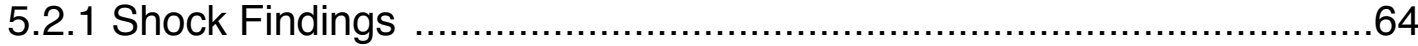

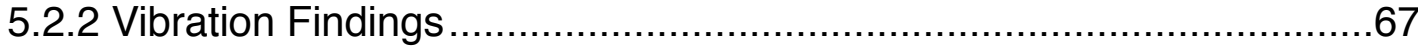

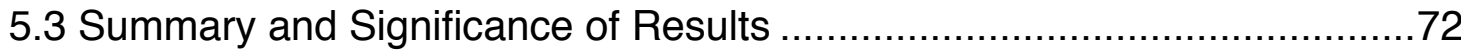

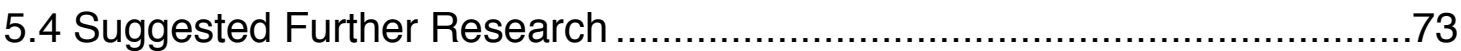

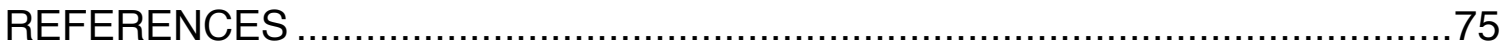




\section{LIST OF TABLES}

Table

1: Seasonal summary analysis of shipments by origin [6] ........................... 11

2: Environmental conditions identified in ASTM D4332-14 [11] ....................... 14

3: Atmospheric pressure level altitudes in next day air shipments [7] .............. 16

4: Altitudes identified in ASTM D6653-01 [13] ............................................. 16

5: Analyzed truck distribution routes [18] ................................................ 24

6: $\mathrm{G}_{\mathrm{rms}}$ levels of different types of trailer suspension [18] ............................. 24

7: Breakpoints of PDS testing curves specific to suspension type [18] ............. 27

8: Overall vibration $\mathrm{G}_{\mathrm{rms}}$ levels observed in delivery vehicles [20]................... 28

9: Composite PDS breakpoints of material handling equipment [21] ................ 30

10: Data recorder setup used for analysis of feeder aircraft [23]...................... 34

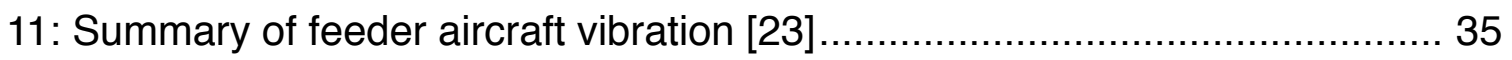

12: Failure rates of varying levels of pressure and vibration [24] ..................... 39

13: E. Lansing, MI / San Luis Obispo, CA distribution shock data [27].............. 42

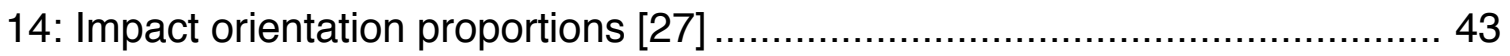

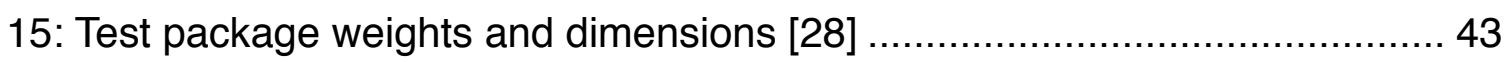

16: Collected domestic drop data [28] ....................................................... 44

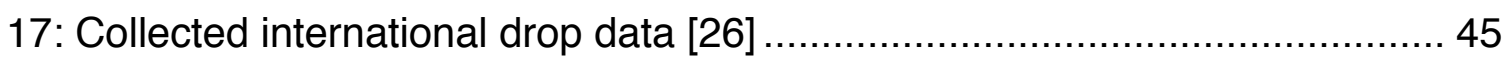

18: Test package weight and dimensions [29] ............................................. 46

19: Proportion of impact type occurrence (\%) [29] ....................................... 47

20: Summary of drop data for small packages [29] ..................................... 48

21: Summary of drop data for medium packages [29] .................................. 49 
22: Summary of drop data for large packages [29]...................................... 50

23: Domestic and International distribution routes investigated ...................... 57

24: Temperature and humidity conditional averages ..................................... 60

25: Minimum / maximum climatic condition levels ....................................... 61

26: Measured drop heights for all air shipment routes................................... 64

27: Highest measured drop heights for all air shipment routes ....................... 65

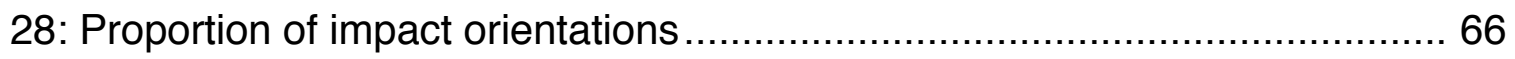

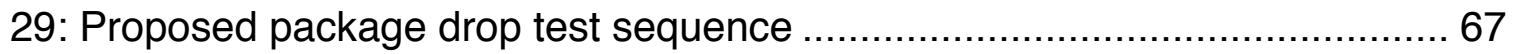

30: Allocation of time spent in various modes of transport ............................... 68

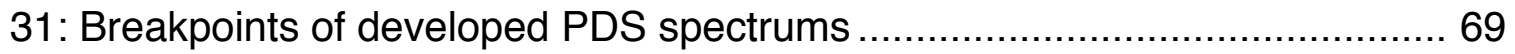




\section{LIST OF FIGURES}

Figure

Page

1: Distribution routes of ground transportation temperature study [6] ............... 10

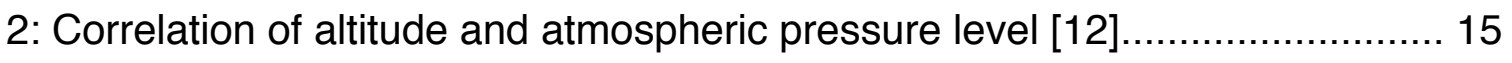

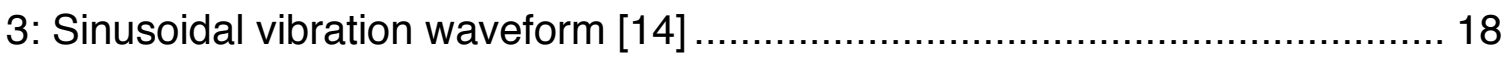

4: Representative PDS plot of truck transport vibration [18].......................... 20

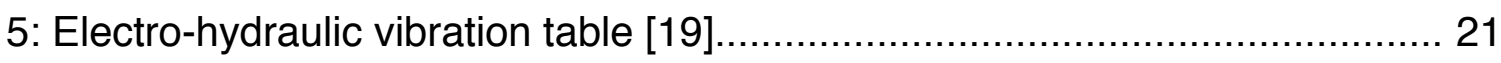

6: Map of analyzed truck distribution routes [18] ...................................... 23

7: Composite vibration profile for air ride suspension trailers [18] .................... 25

8: Composite vibration profile for leaf spring suspension trailers [18] …........... 25

9: Amgen distribution PDS curves before analysis [22] ................................ 31

10: Amgen distribution PDS curves after analysis [22] .................................. 33

11: Cumulative average timer and signal triggered data collected[23] .............. 36

12: Comparative analysis of collected vibration data [23]............................... 37

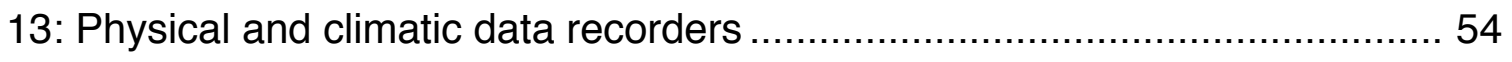

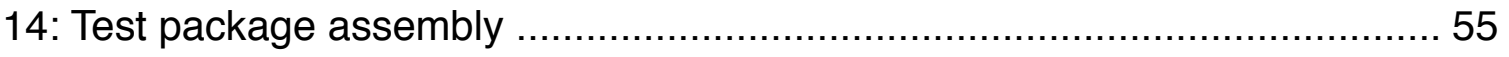

15: Domestic distribution routes investigated ……....................................... 56

16: International distribution routes investigated .......................................... 57

17: Composite PDS spectrum for domestic routes........................................ 70

18: Composite PDS spectrum for international routes..................................... 70

19: Proposed power density spectrum (PDS) test profile ................................ 71 


\subsection{INTRODUCTION}

The modern world is consistently changing into an increasingly global marketplace. Products are often made in one country, stored in a warehouse in another, before finally being shipped to the end recipient. In some cases, the time, location, and other limiting factors warrant the use of air transport to move goods. Transport by air provides the direct benefit of higher speed, greater reliability, and tighter security. This allows the use of a "just in time" inventory system and a great reduction of inventory costs [1].

However, air transport subjects packages to unique levels of physical and climatic distribution hazards. The effect of these hazards should be accounted for throughout the development of a package design to ensure proper performance. In order to best account for these hazards, package performance testing can continually be completed on package design iterations. These performance tests are conducted in an accelerated laboratory environment according to numerous industry accepted standard test methods [2]. The use of testing profiles included in standardized tests allows for the accurate comparison of varying package designs and material options being considered for use.

Although the information provided from standardized package performance tests can be powerful tools to aid the improvement of package designs, the quality of the results from these tests are only as high as the quality of the testing profile 
that is induced. In an effort to improve the accuracy of the testing profiles to allow them to be as truly representative of the hazard levels present in actual distribution, transportation routes are continually studied to further refine the test profiles representing them.

In this study, the complete modern air parcel distribution environment is closely investigated. Data was collected on both the physical hazard levels of vibration and shock, as well as the climatic hazard levels of temperature, humidity, and atmospheric pressure. Furthermore, data was collected from all the different methods of transport experienced between where a package is first dropped off by the sender to where it is finally delivered to the end recipient. After data collection, analysis was performed to develop single, composite testing profiles for each hazard element. These profiles are made to be representative of the "normal conditions" experienced throughout the entire path of distribution. To achieve this objective, data was collected from each mode of transportation used throughout a single distribution route. This includes transport within vehicles such as small delivery vans, large semi trucks, short distance feeder aircraft, and long distance commercial jetliners. It even includes package interaction with sortation and material handling equipment at distribution hubs and movement on carts and dollies at airport facilities. Including data from all these segments of transport 
creates unique testing profiles that are accurately representative of what is experienced throughout the entire distribution environment.

This composite analysis will especially be a significant improvement to the existing vibration profiles. Composite vibration spectrums have not been developed, and therefore not included, in vibration testing requirements as outlined in the US Department of Transportation (DOT) vibration testing requirements for Hazardous Materials, UN vibration testing standards for Dangerous Goods, or the profiles outlined in the ASTM air random vibration test method. For this reason, the development of this single, composite test profile will lead towards a significantly strengthened testing method available for industry wide use. 


\subsection{LITERATURE REVIEW}

\subsection{Air Package Distribution System}

Air package distribution is one of the most commonly used methods to transport goods all throughout the world. According to the World Air Cargo Forecast published by The Boeing Company, although maritime and truck transport could service the same locations for lower cost, air distribution provides transport at a much greater speed and higher reliability [3]. For this reason, air cargo is often used to distribute high value and time sensitive goods, while maritime and truck distribution is commonly used to economically distribute goods of lower value and time sensitivity. As low value goods are transported in much greater quantities, air cargo is only responsible for the distribution of an estimated $1 \%$ of world trade when calculated by tonnage. However, when calculated by the value of goods in transport, air distribution is responsible for a total of $35 \%$ of all world trade [3]. According to the International Air Transport Association, this equates to airlines transporting 52 million metric tons of goods in 2016 [4]. This volume is equal to $\$ 6.8$ trillion worth of goods transported annually, or $\$ 18.6$ billion worth of goods transported every day [4]. Furthermore, although air transport is already a heavily used method of distribution, it has consistently expanded in recent years and is forecasted to continue growing in the future [3].

As air package distribution is such a heavily used method for transport, it is critical the hazard levels commonly experienced throughout the entire multimodal distribution environment are properly identified. Accurate identification of 
these hazards will allow for valuable pre shipment performance testing to be completed to aid in the development of package designs.

In a single route of distribution, a package could be transported within a number of different vehicles and experience the specific distributional hazard levels associated with each. First, the package is transported in a small truck or van to the initial distribution hub. From there, depending on the specific route and location, it could be transferred directly into low altitude feeder aircraft, high altitude commercial jet airliners, or another truck for further ground transport before being included in aircraft transport. Before the package is loaded onto aircraft, it would be transported across the tarmac on a cargo dolly. Once the aircraft arrives at the final destination airport, the package would then move through a truck and van system similar to what was experienced during the initial stages of transport. As a single route of distribution can expose packages to such a wide variety of different hazard levels, the proper identification and integration of these hazards in test profiles is truly necessary for the proper development of an optimal package design.

\subsection{Package Performance Testing}

Some of the most valuable tools used during the development of a new package design is various different types of package performance tests. These tests identify weaknesses in a design, as well as verify package designs will meet required industry specifications set to ensure no excessive harm could be caused throughout the travel of distribution. For example, the US Federal Aviation 
Administration requires that all the packages containing dangerous goods traveling through the US airspace are built to comply with the hazardous materials regulations set forth in the Code of Federal Regulations (49 CFR parts 171-179) [5]. These requirements have been put in place to ensure dangerous goods are properly contained throughout the entire path of distribution.

All package performance tests can be separated into two distinct categories based on the purpose or objective set to be achieved through the completion of the test. Specifically, tests are performed in order to quantify a certain aspect of the design, or to evaluate the performance of a design when subjected to one of the various different physical or climatic hazards that could be encountered throughout distribution.

The first type of package performance test quantifies the performance of a certain element included in a package design. This could include material characterization or the analysis of a particular component included. Oftentimes, these tests are performed during the initial developmental stages of a new design as they identify optimal features to include. Some common examples of quantification type testing like this are listed below [2].

- Tensile testing

- Compression testing

- Burst testing

- Scuff testing

- Edge crush testing (ECT)

- Peel strength testing 
The second main category of performance tests create the peak levels of a certain distribution hazard. These tests could be used to directly compare the performance of different design iterations or validate a package design will properly perform before being released for full scale production. Simulation testing like this is a very valuable tool as it can save substantial amounts of time and money through the identification of flaws before widespread packaging or product damage is incurred. Many simulation type tests for different hazard elements have been developed. Some specific examples of commonly performed simulation tests are listed below [2].

- Atmospheric conditioning (Temperature, humidity, and atmospheric pressure)

- Vibration testing

- Drop (shock) testing

- Compression testing

These peak hazard level simulation tests are conducted in a controlled laboratory environment to allow for the direct comparison of varying design iterations and materials being considered for use. In order for the tests being performed to be as repeatable as possible, there are several organizations that develop and publish testing standards that specify the exact procedure and hazard testing levels. By maintaining adherence to these testing standards, the tests can be performed with a high level of repeatability between different test samples and testing locations. Some of the most prominent organizations that develop and publish package performance testing standards include: 
- American Society of Testing and Materials (ASTM)

- International Safe Transit Association (ISTA)

- International Standards Organization (ISO)

- European Committee for Standardization (CEN)

- Technical Association of Pulp and Paper Industry (TAPPI)

- United States Code of Federal Regulations (CFR)

Details of the testing that has been developed for the physical hazards of vibration, and shock, as well as testing developed for the climatic hazard levels of temperature, humidity, and atmospheric pressure, are included in the sections describing each of these hazards below.

\subsection{Climatic Distribution Hazards}

Throughout distribution, packages will be exposed to a wide variety of different environmental conditions. The most commonly experienced include varying levels of temperature, humidity, and atmospheric pressure. Each of these conditions have the ability, given the proper level of severity, to induce damage to both the packaging and products within. Many combinations of conditional extremes, fluctuations, and extended durations could lead to damage being incurred.

\subsubsection{Temperature and Humidity}

One of the most easily identifiable climatic conditions that should be considered during package design is varying levels of temperature. Not only could temperature extremes induce cosmetic damages, such as material wrinkling and color change, but package function could also be compromised in the form of 
adhesive failure and a reduction in compression strength. For this reason, some transportation vehicles have been built with engineering controls to maintain their internal temperature at levels that will not impose damage, but it is common that vehicles do not control the levels present within them.

As packages travel through the path of distribution, numerous temperature levels could be experienced for varying durations of time. Many of these temperatures have been identified in recent studies, and the findings of some of them are expanded upon in the sections below.

In one study, completed by researchers at the Rochester Institute of Technology, the ambient temperatures experienced in the ground transportation environment throughout the United States were analyzed in depth [6]. In this study, temperature data was collected in the distribution routes of a large pharmaceutical company. It included routes between the western, eastern, and southern distribution centers and 19 different receiving locations spread all across the country. The wide range of distribution routes included in this study is easy to see in the map shown in figure 1. 


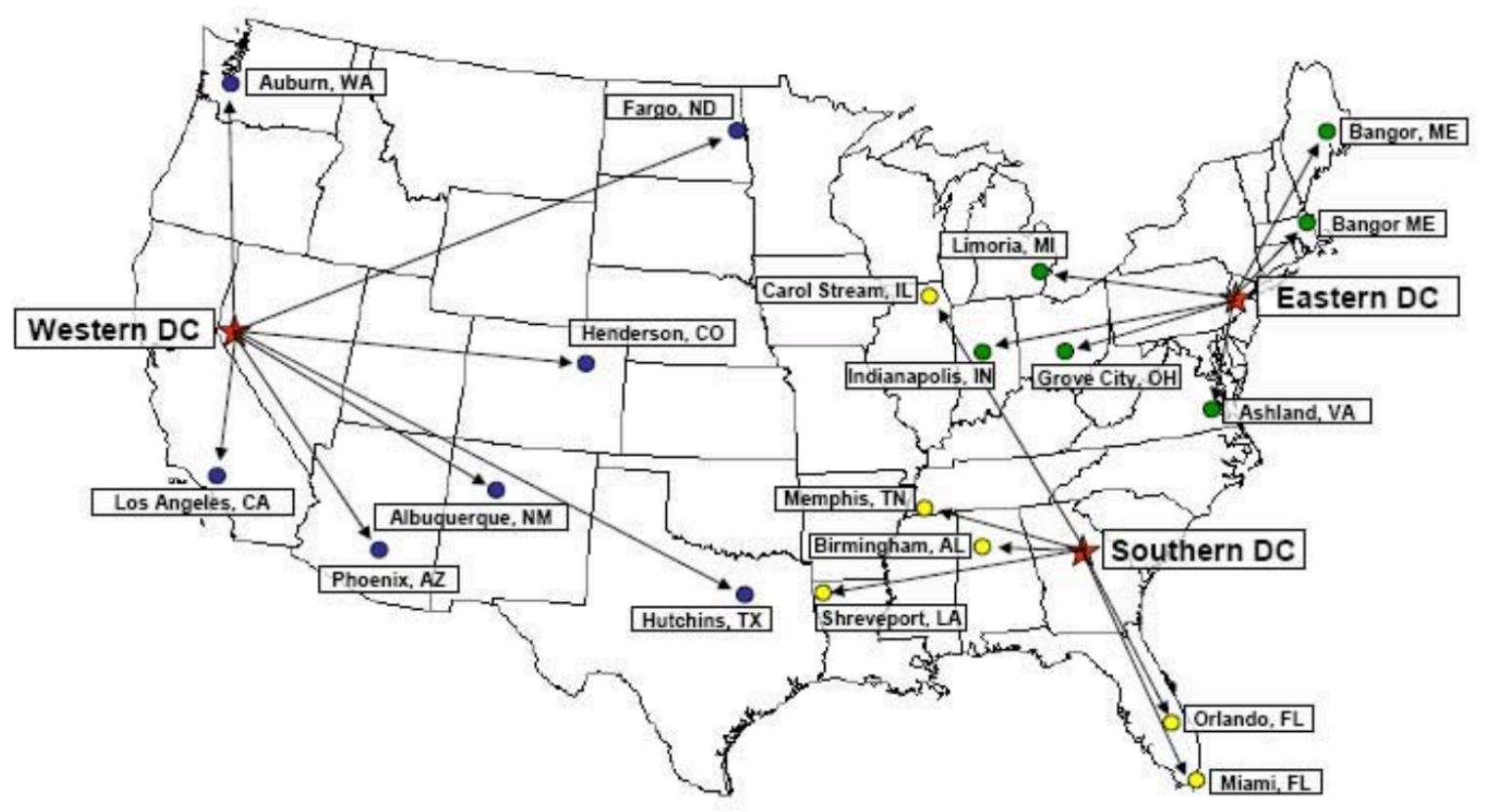

Figure 1: Distribution routes of ground transportation temperature study [6]

In addition to covering a vast majority of different areas throughout the United States, data was collected during all four seasons. For this reason, this is considered a viable summary of the temperatures experienced in the domestic ground shipping environment. At the conclusion of this study, temperatures ranging from 9.1 to $60.9^{\circ} \mathrm{C}$ during the warmest season, and between -19.8 and $46.1^{\circ} \mathrm{C}$ in the coolest season were gathered. This study found more severe temperature conditions were experienced than is claimed by the United Parcel Service and is currently integrated into the ASTM temperature testing profile. The minimum, maximum, and mean temperatures experienced in each area of distribution and during each season is presented in table 1. 
Table 1: Seasonal summary analysis of shipments by origin [6]

\begin{tabular}{|c|c|c|c|c|c|}
\hline \multirow{4}{*}{ Origin } & $\begin{array}{c}\text { Number of } \\
\text { shipments }\end{array}$ & Season & Mean $\left({ }^{\circ} \mathbf{C}\right)$ & Minimum $\left({ }^{\mathbf{C}} \mathbf{C}\right)$ & Maximum $\left({ }^{\circ} \mathbf{C}\right)$ \\
\hline \multirow{3}{*}{ Eastern DC } & \multirow{3}{*}{170} & Summer & 23.3 & 10.1 & 46.0 \\
\cline { 3 - 6 } & & Winter & 7.2 & -11.5 & 28.1 \\
\cline { 3 - 6 } & & Fall & 16.4 & -7.2 & 34.7 \\
\cline { 3 - 6 } & \multirow{3}{*}{ Western DC } & Spring & 14.9 & -1.9 & 36.4 \\
\hline \multirow{3}{*}{174} & Summer & 27.4 & 9.1 & 60.9 \\
\cline { 3 - 6 } & & Winter & 5.0 & -19.8 & 46.1 \\
\cline { 3 - 6 } & & Fall & 16.6 & -6.6 & 38.9 \\
\cline { 3 - 6 } & & Spring & 16.2 & -3.1 & 38.3 \\
\hline \multirow{3}{*}{ Southern DC } & \multirow{3}{*}{150} & Summer & 29.1 & 17.6 & 45.6 \\
\cline { 3 - 6 } & & Winter & 12.7 & -10.8 & 29.9 \\
\cline { 3 - 6 } & & Fall & 20.6 & -2.7 & 45.9 \\
\cline { 3 - 6 } & & Spring & 19.7 & 3.3 & 36.4 \\
\hline
\end{tabular}

In a different study, completed in 2010 by researchers at California Polytechnic State University, the temperatures present in the domestic next day air shipment environment were identified [7]. By sending small instrumented test packages between East Lansing, MI and the two receiving locations of Twin Falls, ID and San Luis Obispo, CA, the temperature values present within both large airliners and smaller feeder aircraft were identified.

At the conclusion of the study, temperatures ranging from 7.8 to $32^{\circ} \mathrm{C}$ were found inside feeder aircraft, and from 11.5 to $29.2^{\circ} \mathrm{C}$ inside high altitude aircraft. Also, the temperatures experienced in high altitude air shipments were less 
variable as these aircraft are better insulated so there is more consistent temperatures present [7].

In a study completed in 2001 by the United Parcel Service (UPS), the conditions a package could experience in the single parcel shipping environment were identified and the findings were presented to the American Society of Testing and Materials (ASTM) [8]. From this study, it was found that the temperature levels were typically maintained at approximately 20 to $23^{\circ} \mathrm{C}$ in cargo air jets, but they could range anywhere from -4 to $24^{\circ} \mathrm{C}$ in feeder aircraft. While packages are transported on the ground, temperature extremes ranging from -15 to $30^{\circ} \mathrm{C}$ and average temperatures ranging from -4 to $18^{\circ} \mathrm{C}$ could be experienced. These findings have been accepted and incorporated by the American Society of Testing and Materials in the testing standards they have published [8].

Humidity, or the amount of water vapor present in the air, is another climatic condition that is always being experienced to varying degrees throughout package distribution. Humidity can have significant effect on both the cosmetics and functional performance of a package. As the humidity levels present within a vehicle will adjust in correspondence to the wide array of varying humidity levels present in different geographic areas, it is of much importance to identify and adjust specific testing protocol in correspondence to the conditions that could be experienced in a particular distribution route.

The potential damage types caused through various levels of temperature and humidity are greatly dependent on the type of material included in a package 
design. Different grades of plastic are highly susceptible to damage from high temperature levels, whereas humidity has a greater damaging effect on the paper components included in a design $[9,10]$. When exposed to high temperature levels, different plastic compounds will incur a reduction in structural strength or material deformation. More specifically, a higher temperature will lead to a reduction in elastic modulus and a reduction in tensile and compression strength [9]. Not only could this end in damage to a single package, but it could also lead to a stacking collapse and the possible harm of additional packages. As paper is hygroscopic in nature, higher levels of humidity will readily lead to the absorption of moisture by paper components. This moisture absorption will lead to de-lamination of some types of adhesives and a disruption of hydrogen bonds in the paper fibers leading to a reduction in structural strength of the material itself [10].

To account for different temperature and humidity conditions, the American Society of Testing and Materials has published ASTM D4332: Standard Practice for Conditioning Containers, Packages, or Packaging Components for Testing. The procedure to complete ASTM D4332-14 specifies for the test specimens to be conditioned in the environment that will be experienced for a duration long enough for the test specimen to completely reach equilibrium with the conditions that are present. Once the test specimens have reached equilibrium, any following physical performance test should be performed, if possible, while the specimen is still within the actual conditions. If this is not possible, any additional 
testing should be immediately performed after the removal of the test specimen from the conditioning chamber.

When referencing ASTM D4332-14, temperature and humidity conditions are presented for seven different climates. These conditions, paired with their corresponding climates, are presented in table 2 below.

Table 2: Environmental conditions identified in ASTM D4332-14 [11]

\begin{tabular}{|l|c|c|c|}
\hline \multirow{2}{*}{\multicolumn{1}{c|}{ Climatic Condition }} & \multicolumn{2}{c|}{ Temperature } & Relative Humidity \\
\cline { 2 - 4 } & ${ }^{\circ} \mathbf{F}$ & ${ }^{\circ} \mathbf{C}$ & $\%$ \\
\hline Cryogenic & $-55 \pm 3$ & $-67 \pm 6$ & $\cdots$ \\
\hline Extreme Cold & $-30 \pm 2$ & $-22 \pm 4$ & $\cdots$ \\
\hline Frozen Food Storage & $-18 \pm 2$ & $0 \pm 4$ & $\cdots$ \\
\hline Refrigerated Storage & $5 \pm 2$ & $41 \pm 4$ & $85 \pm 5$ \\
\hline Temperate High Humidity & $20 \pm 2$ & $68 \pm 4$ & $90 \pm 5$ \\
\hline Tropical & $40 \pm 2$ & $104 \pm 4$ & $90 \pm 5$ \\
\hline Desert & $60 \pm 2$ & $140 \pm 4$ & $15 \pm 5$ \\
\hline
\end{tabular}

\subsubsection{Atmospheric Pressure}

Another climatic condition that must be properly accounted for during package design is the varying levels of atmospheric pressure that will be experienced throughout a route of distribution. As packages are a packed and sealed at ground level, great stress could be induced near any contained areas of space in the package when elevated to altitudes with the conditions of lower atmospheric pressure. The effects of the pressure differential can easily be seen in thin walled plastic bottles and film material bags. If the stress caused by the differential in 
pressures is greater than the material is able to support, tearing and material deformation could occur.

At sea level, atmospheric pressure is $14.7 \mathrm{PSI}$. As altitude is gained during air transport or while being included in ground transport over a tall mountain pass, air pressure will reduce. The rate of pressure reduction in correspondence to a rise in altitude is graphically displayed in figure 2 . The findings of pressure levels experienced in different types of transportation is discussed below.

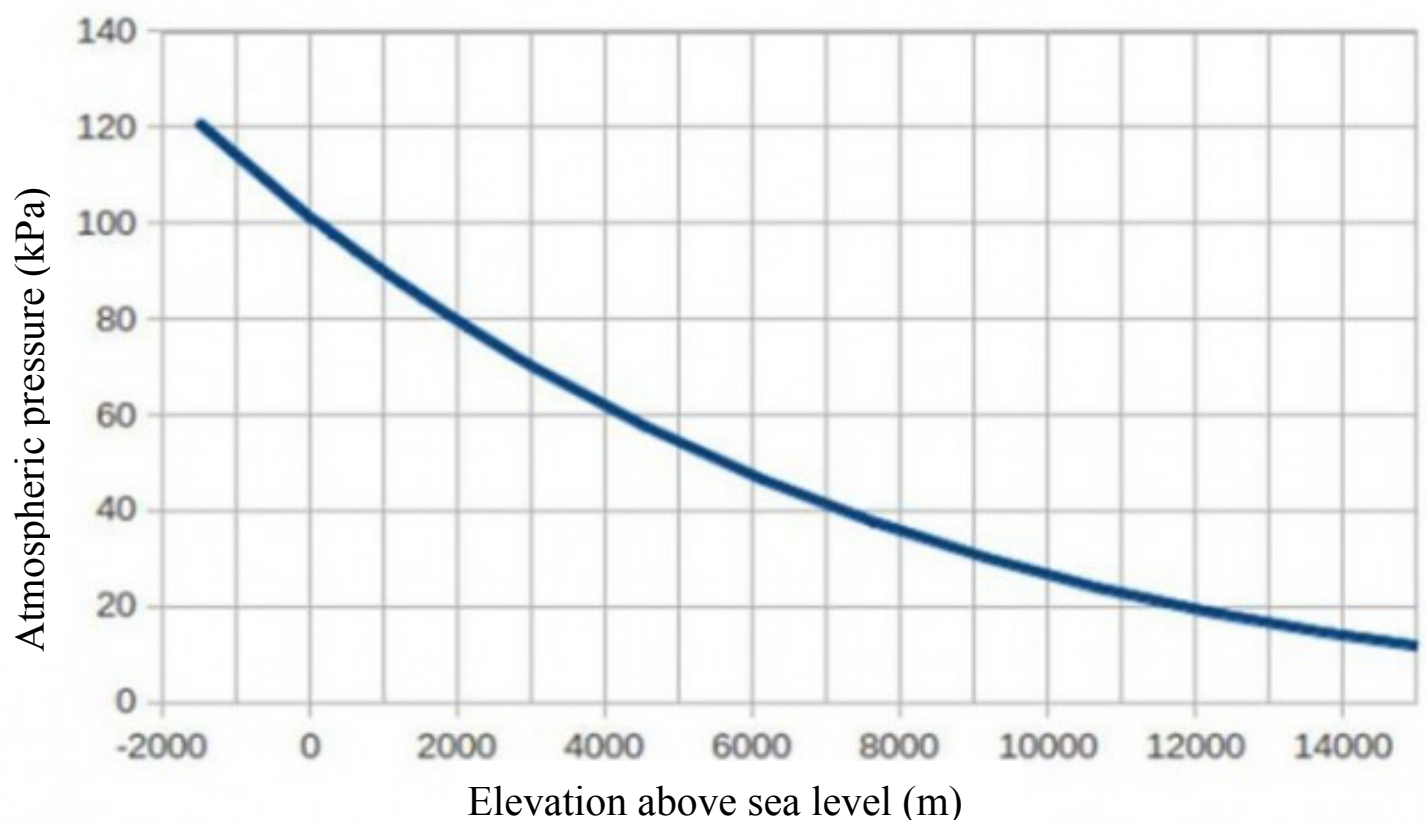

Figure 2: Correlation of altitude and atmospheric pressure level [12]

In a recent study of the next day air shipping environment, the pressure levels experienced in both feeder aircraft and high altitude commercial jetliners were investigated. To complete this study, test packages were sent between East Lansing, Ml and the two receiving locations of Twin Falls, ID and San Luis 
Obispo, CA. The pressure levels found at the completion of this study are included in table 3 below [7].

Table 3: Atmospheric pressure level altitudes in next day air shipments [7]

\begin{tabular}{|c|c|}
\hline Type of Transport & Experienced Pressure Level Altitude \\
\hline $\begin{array}{c}\text { Pressurized High Altitude } \\
\text { Cargo Air Jets }\end{array}$ & $835-2168 \mathrm{~m}$ \\
\hline $\begin{array}{c}\text { Non-pressurized } \\
\text { Feeder Aircraft }\end{array}$ & $2616-5320 \mathrm{~m}$ \\
\hline
\end{tabular}

The pressure levels identified in the currently published ASTM test standard (Standard Test Methods for Determining the Effects of High Altitude on Packaging Systems by Vacuum Method (ASTM D6653-01)), are presented below in table 4 [13].

Table 4: Altitudes identified in ASTM D6653-01 [13]

\begin{tabular}{|c|c|}
\hline Type of Transport & Experienced Pressure Level Altitude \\
\hline $\begin{array}{c}\text { Pressurized High Altitude } \\
\text { Cargo Air Jets }\end{array}$ & $2438 \mathrm{~m}$ \\
\hline $\begin{array}{c}\text { Non-pressurized } \\
\text { Feeder Aircraft }\end{array}$ & $\begin{array}{c}\text { Typical Altitudes: } 3963-4877 \mathrm{~m} \\
\text { Highest observed altitudes: } 6017 \mathrm{~m}\end{array}$ \\
\hline Ground Transport & $3,658 \mathrm{~m}$ \\
\hline
\end{tabular}

After a comparison, it can be seen the pressure levels observed in the two studies noted above are in agreement with each other. In addition, the pressure change rates and duration of flights also fell into accordance with one another. For this reason, the atmospheric pressure conditions identified in ASTM D6653-01 are considered to be accurately representative of the conditions that 
could be experienced in aircraft distribution. To conduct ASTM D6653-01, the following procedure is conducted.

1. Place the test specimen(s) in the chamber.

2. Close and reduce pressure in chamber at a rate of $305 \mathrm{~m}(1,000 \mathrm{ft})$ every $30-60$ secs to the desired pressure level customized per the route of distribution.

3. Maintain this vacuum for 60 mins.

4. At the end of 60 mins, increase pressure in chamber at the rate of $305 \mathrm{~m}$ $(1,000 \mathrm{ft})$ every $30-60$ secs.

5. Remove tested samples and conduct inspection for damage present.

\subsection{Physical Distribution Hazards}

Just as various climatic hazards are experienced throughout any distribution route, many physical hazards are also encountered. The most common include vibration and shock.

\subsubsection{Vibration}

Vibration is experienced in every distribution route to varying degrees. As it can contribute to significant packaging and product damage, it is crucial to properly account for it during package design.

The most basic form of vibration is sinusoidal vibration. It is the consistent oscillatory movement made by an object in relation to another object at a single frequency. Due to its simplicity, it can be defined through the level of amplitude 
and the frequency of its movement [14]. A representative example of a sinusoidal waveform is shown in figure 3.

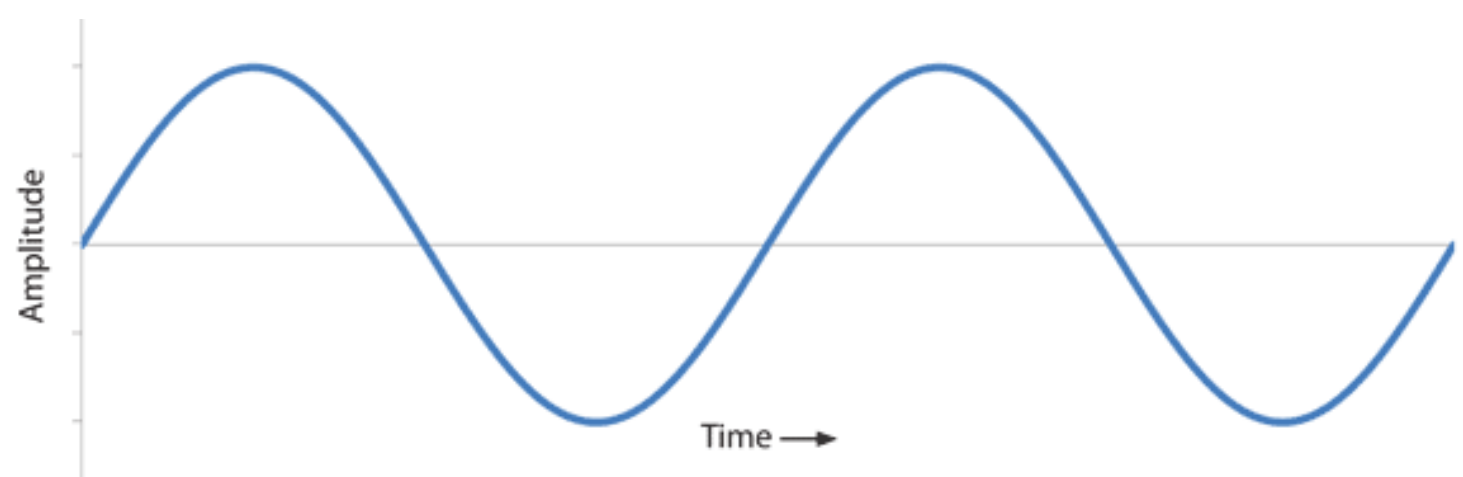

Figure 3: Sinusoidal vibration waveform [14]

As sinusoidal vibration does not occur often in actual distribution, it should not be used as a form of validation testing. However, there are developed test methods that do employ it. One such method is known as a package resonance search and dwell test [15]. In this test, the test package is loosely loaded (not vertically restrained) on the vibration table and the frequency is consistently and gradually increased in order to determine the natural resonant frequency. This frequency level can be identified as the point of greatest induced excitation. When the complete package is under investigation, this frequency is identified through easily slipping a $2 \mathrm{~mm}$ shim underneath the package. When a specific component of the package is under investigation, the method used to identify the frequency that is inducing the greatest excitation must be identified before conducting the test. Once the resonant frequency is identified for the element under investigation, the vibration test remains to induce this resonant frequency 
for a specified time period. This test allows quick performance comparison of varying package designs as it continuously subjects the packages under test to only the most damaging vibration frequencies.

The more complex type of vibration commonly experienced during distribution is random vibration. This vibration consist of numerous different waveforms, each occurring simultaneously with one another [16]. As these waveforms are sourced from multiple locations throughout a vehicle, they are all occurring at unique frequencies and power density levels. For example, during truck transport, high frequency vibration can be internally sourced from the vehicles engine, while lower frequency vibration can be externally sourced from the truck tires and suspension reacting to the conditions of the road. Not only could each vibration contribute to varying levels of damage by themselves, but when they are experienced together, completely new types of damage could be created [16]. Furthermore, the occurrence rate of each waveform is in consistent adjustment throughout the entire route of distribution. Due to random vibration being of much greater complexity, it has a much more complicated method of quantification associated with it.

Random vibration is quantified through creating a power density spectrum (PDS) breakpoint curve. To create this curve, the strength of each frequency of vibration is identified [17]. This strength, or power density (PD) level, is defined in the terms of the mean-square magnitude of acceleration per unit bandwidth $\left(\mathrm{G}^{2} / \mathrm{Hz}\right)$. Once identified, the PD level and frequency breakpoints are plotted in relation to 
one another and a PDS breakpoint curve is created. This curve identifies the distribution of power across the entire range of frequencies present. When the PDS breakpoint curve is added to the PDS plot, the strength of the entire vibration event can be quantified by identifying the root mean square of the acceleration levels of all the waveforms present in a particular event of vibration. This $\mathrm{G}_{\mathrm{rms}}$ level can be identified as the total area underneath the PDS breakpoint curve. A representative example of a PDS plot is shown below in figure 4 [17].

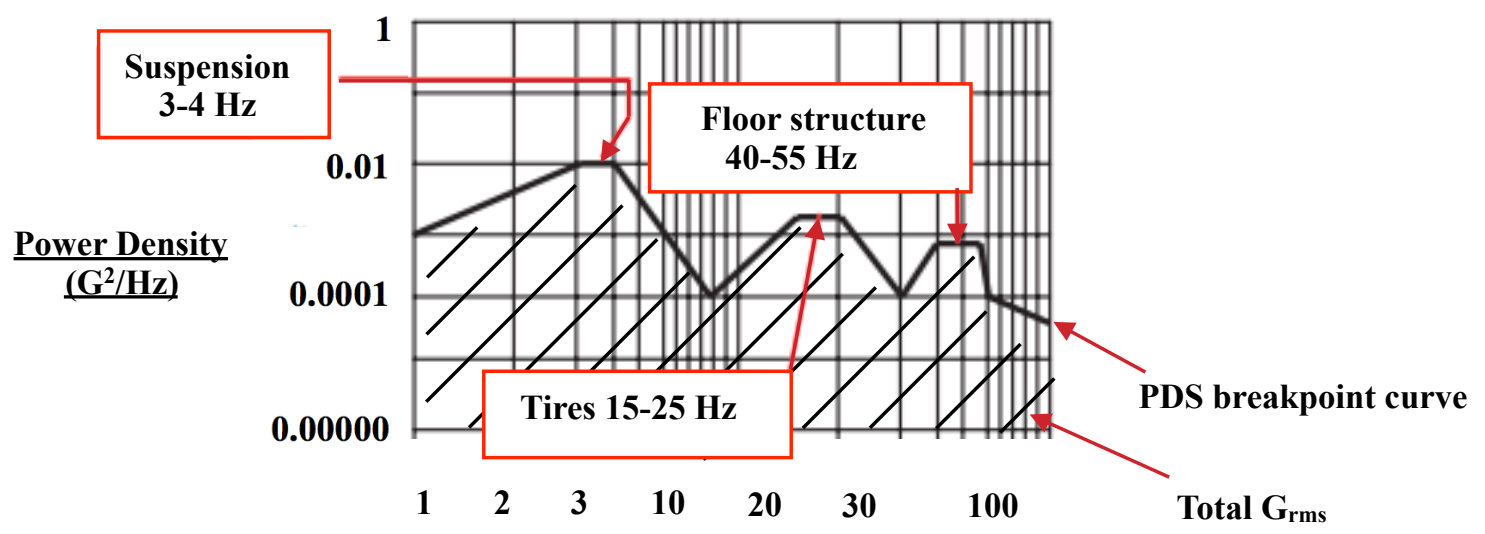

Frequencv (Hz)

Figure 4: Representative PDS plot of truck transport vibration [18]

As varying levels of random vibration are experienced in all distribution routes, and it leads to such a high damaging potential, it is critical vibration related abuse is considered during the design of a package. In order to reduce the amount of time and resources required to achieve this, accelerated vibration simulation tests are conducted in laboratory settings on electro-hydraulic vibration tables 
(figure 5). These vibration tables are capable of recreating vibration with the same distribution of power across various frequencies as is defined in the PDS curve representing a particular event of vibration.

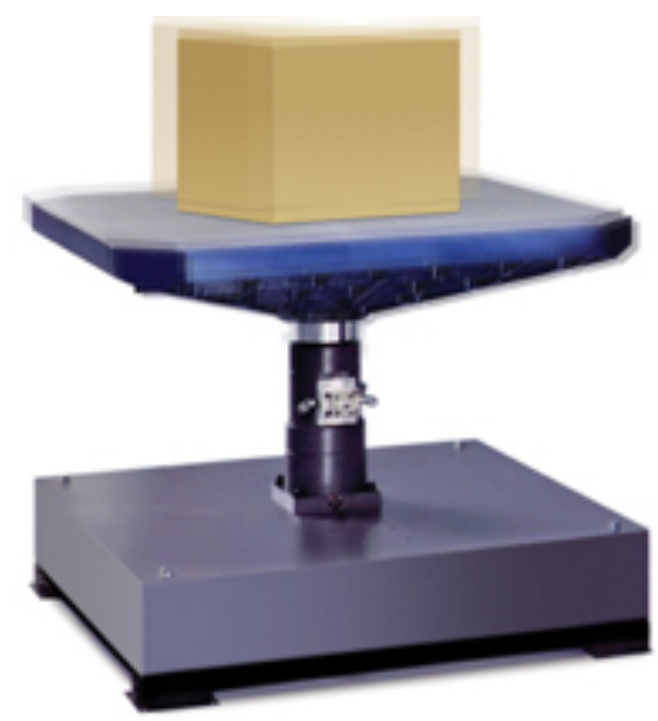

Figure 5: Electro-hydraulic vibration table [19]

As these simulation tests are directly based off of a PDS breakpoint curve (profile), they are only as accurately representative of actual distribution as the accuracy of the PDS profile included within them. For this reason, studies are continually being completed to further develop known PDS profiles.

To collect data for the development of a PDS profile, electronic data recorders are either directly mounted to transport vehicles or positioned within a test package being transported through a particular distribution route of interest. When the data recorders are mounted on transport vehicles, direct input vibration levels are recorded. As this allows for the collection of input vibration where no 
energy has been attenuated by packaging, this is the preferred setup for data collection. When it is not possible to achieve this data recording setup, it is critical the test packages are designed to limit the amount vibration force attenuation as much as possible so there is little deviation between the true input vibration and the vibration being experienced by the data recorders mounted inside test packages.

Through the use of data recorders, vibration analysis can be performed on a wide array of possible variables encountered during distribution. The scope of a specific investigation like this could range from being as narrow and focused as the analysis of the vibration experienced during transport in a specific section of a particular distribution route, to as broad and encompassing as the identification of the overall vibration that is experienced throughout a complete route of distribution. The results of analyses like this can be used to update the PDS vibration profiles included in existing performance tests and allow a more truly representative vibration simulation test to be performed. Many such studies have been performed in recent years. Some of these studies are discussed in the sections below.

In one study, researchers from California Polytechnic State University, Michigan State University, and Lansmont Corporation investigated the effect of different types of trailer suspension on the vibration levels experienced [18]. To complete this study, researchers collected and compared the vibration levels on leaf spring and air ride suspension semi truck trailers. To characterize this specific aspect of 
distribution, the test packages, each containing a Lansmont SAVER data recorder, were sent on 14 different routes of distribution and analyzed over $16,000 \mathrm{~km}$ of domestic truck transport. A map with all the included distribution routes is shown below in Figure 6 . The origin and receiving locations, as well as the distances and suspension types used in each route, are identified in table 5 [18].

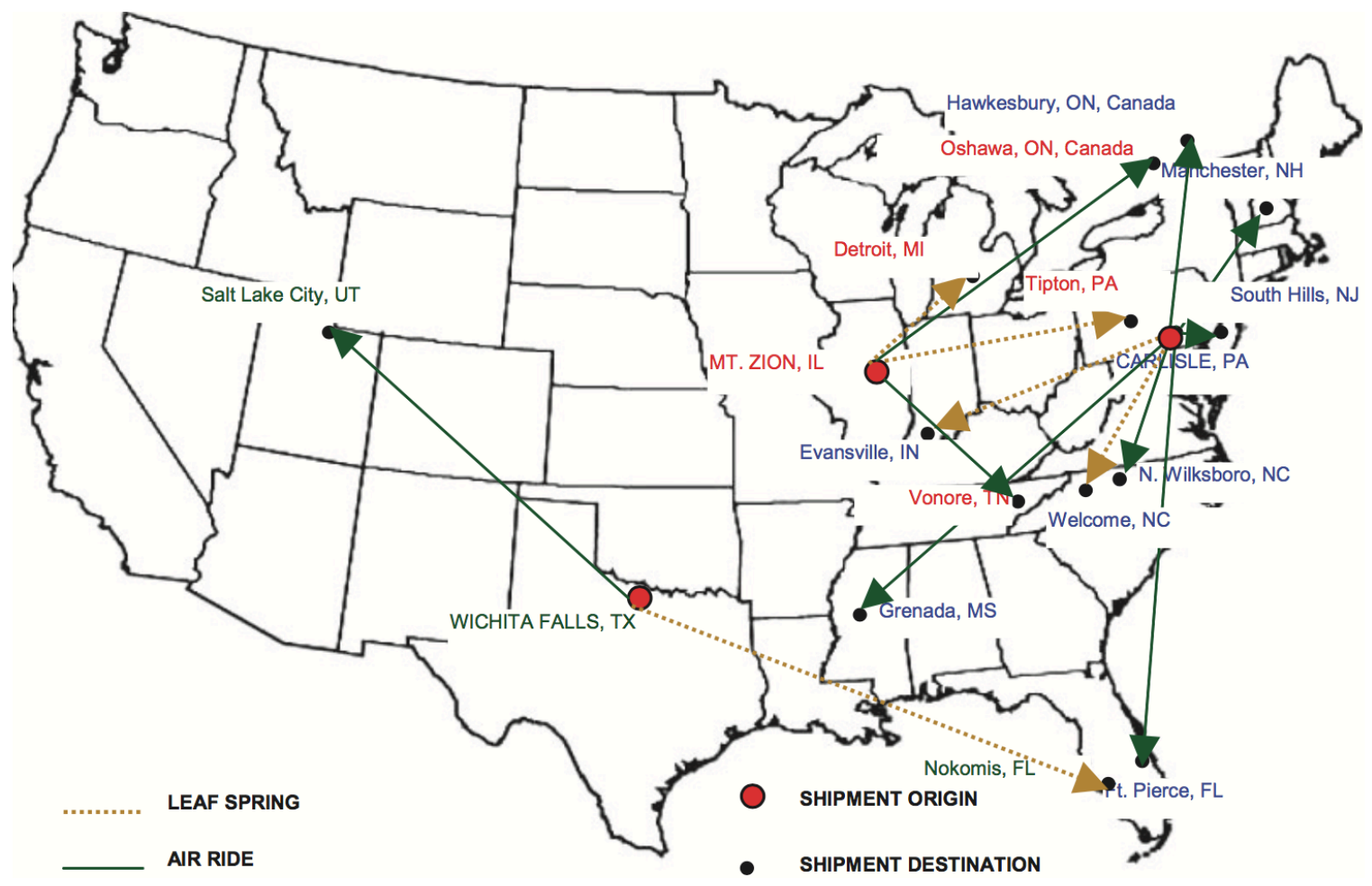

Figure 6: Map of analyzed truck distribution routes [18] 
Table 5: Analyzed truck distribution routes [18]

\begin{tabular}{|c|c|c|c|c|}
\hline Trip \# & Distance (Miles) & Suspension Type & Route Origin & Route Destination \\
\hline $\mathbf{1}$ & 430 & Leaf spring & Carlisle, PA & Welcome, NC \\
\hline $\mathbf{2}$ & 680 & Leaf spring & Carlisle, PA & Evansville, IN \\
\hline $\mathbf{3}$ & 445 & Air ride & Carlisle, PA & Manchester, NH \\
\hline $\mathbf{4}$ & 200 & Air ride & Carlisle, PA & South Hills, NJ \\
\hline $\mathbf{5}$ & 400 & Air ride & Carlisle, PA & North Wilkesboro, NC \\
\hline $\mathbf{6}$ & 490 & Air ride & Carlisle, PA & Hawkesbury, ON \\
\hline $\mathbf{7}$ & 1050 & Air ride & Carlisle, PA & Ft. Pierce, FL \\
\hline $\mathbf{8}$ & 1135 & Air ride & Carlisle, PA & Grenada, MS \\
\hline $\mathbf{9}$ & 1245 & Leaf spring & Wichita Falls,TX & Nokomis, Florida \\
\hline $\mathbf{1 0}$ & 1332 & Air ride & Wichita Falls,TX & Salt lake city, UT \\
\hline $\mathbf{1 1}$ & 625 & Leaf spring & Mt. Zion & Tipton PA \\
\hline $\mathbf{1 2}$ & 680 & Air ride & Mt. Zion & Oshawa, ON \\
\hline $\mathbf{1 3}$ & 625 & Air ride & Mt. Zion & Carlex, Vonore \\
\hline $\mathbf{1 4}$ & 425 & Leaf spring & Mt. Zion & Detroit, MI \\
\hline
\end{tabular}

After an analysis of the collected vibration data, it was found the vibration levels were significantly higher in the leaf spring trailers when compared to the air ride suspension trailers. All the data was compiled into composite PDS plots (figure $7,8)$ and the $\mathrm{G}_{\mathrm{rms}}$ values identified are presented below in table 6 .

Table 6: $\mathrm{G}_{\mathrm{rms}}$ levels of different types of trailer suspension [18]

\begin{tabular}{|c|c|c|}
\hline Trip \# & $\mathbf{3 0 \%}$ High Grms & $\mathbf{7 0 \%}$ Low Grms \\
\hline Leaf spring suspension & 0.659 & 0.354 \\
\hline Air ride suspension & 0.283 & 0.146 \\
\hline
\end{tabular}




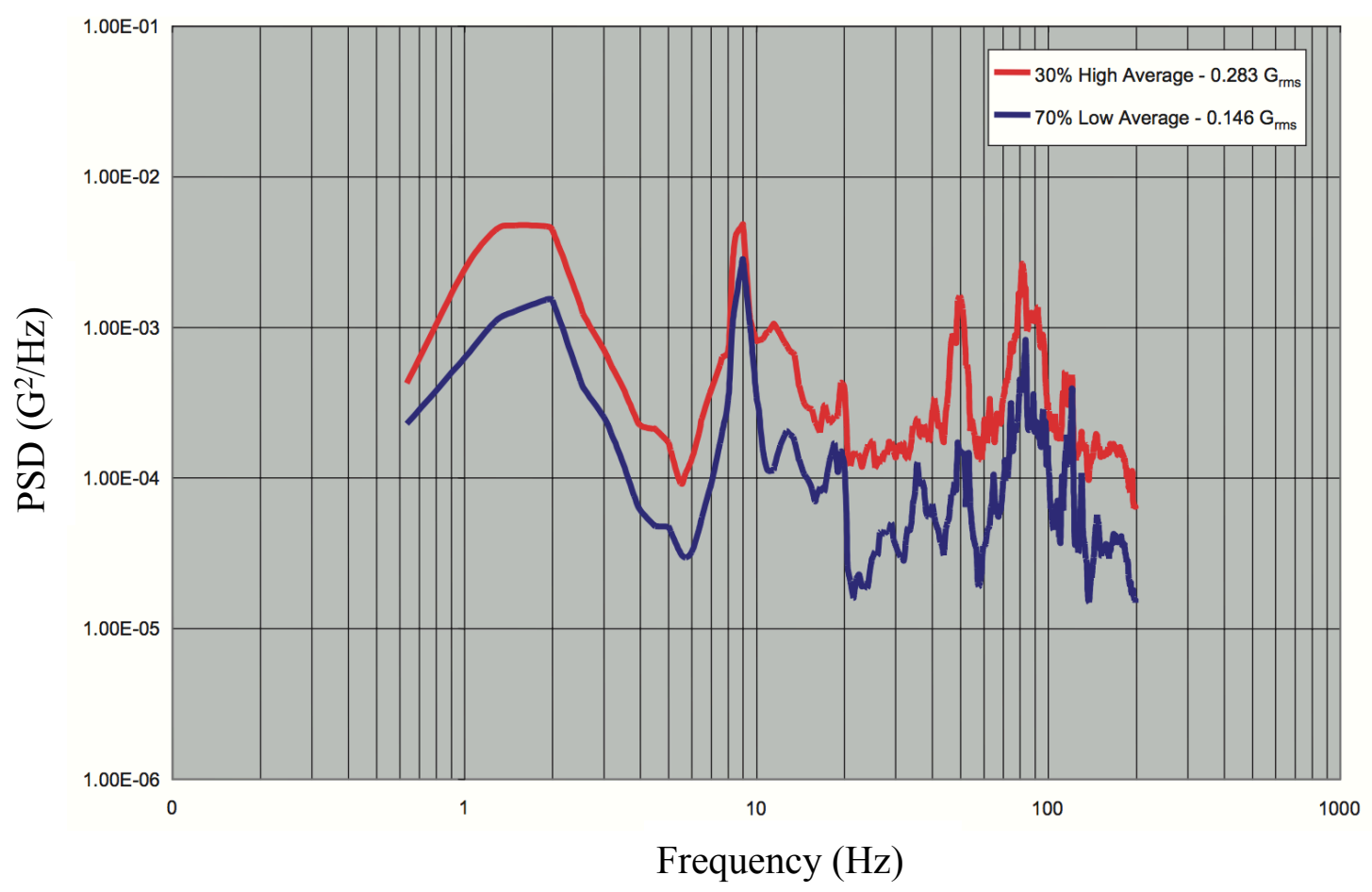

Figure 7: Composite vibration profile for air ride suspension trailers [18]

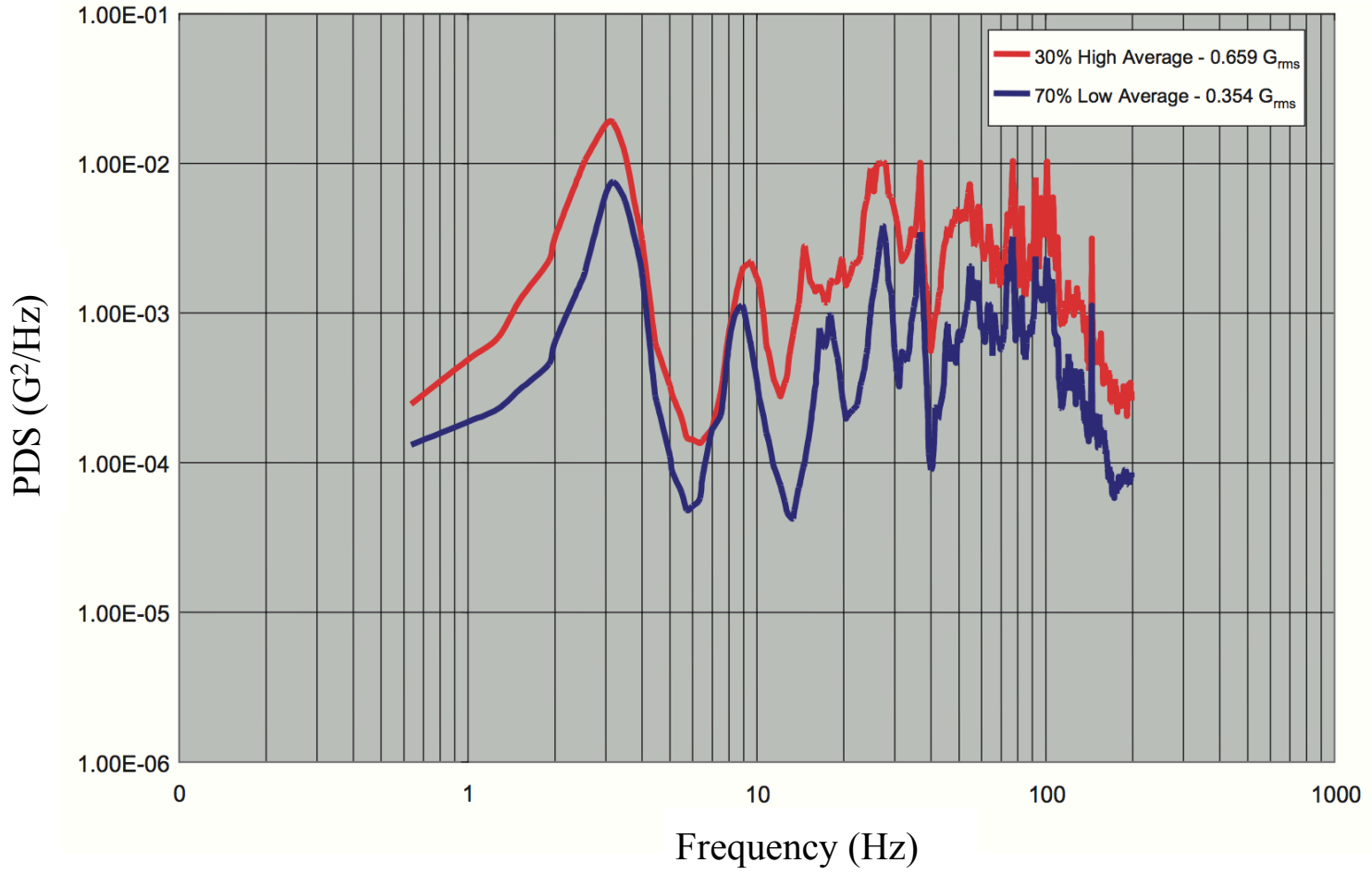

Figure 8: Composite vibration profile for leaf spring suspension trailers [18] 
After collecting these values, test profiles representing each type of suspension were developed. These test profiles are designed to be run in accordance with ASTM D4728. For each type of suspension, the collected data was grouped by the $30 \%$ highest levels and $70 \%$ lowest level of vibration. The test time was split to run the $70 \%$ low level profile for 60 minutes and the $30 \%$ high level profile for $30 \mathrm{~min}$. The high and low level test profile breakpoints for each suspension type can be found in table 7 below. The breakpoints of these PDS curves can be entered into testing machinery to simulate this level of vibration. 
Table 7: Breakpoints of PDS testing curves specific to suspension type [18]

\begin{tabular}{|c|c|c|c|c|c|}
\hline \multicolumn{3}{|c|}{$\begin{array}{l}\text { Air Ride Suspension } \\
\text { Testing Spectra Breakpoints }\end{array}$} & \multicolumn{3}{|c|}{$\begin{array}{l}\text { Leaf Spring Suspension } \\
\text { Testing Spectra Breakpoints }\end{array}$} \\
\hline Frequency $(\mathrm{Hz})$ & $\begin{array}{l}70 \% \text { Low } \\
\text { Power } \\
\text { Density } \\
\left(G^{2} / \mathbf{H z}\right)\end{array}$ & $\begin{array}{l}\text { 30\% High } \\
\text { Power } \\
\text { Density } \\
\left(\mathbf{G}^{2} / \mathrm{Hz}\right)\end{array}$ & Frequency $(\mathrm{Hz})$ & $\begin{array}{l}70 \% \text { Low } \\
\text { Power } \\
\text { Density } \\
\left(\mathbf{G}^{2} / \mathbf{H z}\right)\end{array}$ & $\begin{array}{c}\text { 30\% High } \\
\text { Power } \\
\text { Density } \\
\left(G^{2} / \mathrm{Hz}\right)\end{array}$ \\
\hline 1 & 0.00242 & 0.00062 & 1 & 0.00048 & 0.00018 \\
\hline \multirow{2}{*}{2} & \multirow{2}{*}{0.00446} & \multirow{2}{*}{0.00152} & 2 & 0.00319 & 0.00063 \\
\hline & & & 3 & 0.01820 & 0.00625 \\
\hline 6 & 0.00013 & 0.00003 & 6 & 0.00014 & 0.00005 \\
\hline 9 & 0.00483 & 0.00282 & 9 & 0.00202 & 0.00108 \\
\hline 10 & 0.00081 & 0.00034 & 10 & 0.00028 & 0.00007 \\
\hline 20 & 0.00039 & 0.00012 & 20 & 0.00195 & 0.00024 \\
\hline 30 & 0.00013 & 0.00003 & 30 & 0.00482 & 0.00058 \\
\hline 40 & 0.00027 & 0.00006 & 40 & 0.00057 & 0.00009 \\
\hline 50 & 0.00158 & 0.00015 & 50 & 0.00430 & 0.00075 \\
\hline 60 & 0.00019 & 0.00003 & 60 & 0.00296 & 0.00081 \\
\hline 70 & 0.00034 & 0.00009 & 70 & 0.00161 & 0.00057 \\
\hline 80 & 0.00159 & 0.00045 & 80 & 0.00153 & 0.00073 \\
\hline 90 & 0.00117 & 0.00020 & 90 & 0.00177 & 0.00081 \\
\hline 100 & 0.00028 & 0.00012 & 100 & 0.00432 & 0.00159 \\
\hline 200 & 0.00006 & 0.00001 & 200 & 0.00026 & 0.00008 \\
\hline
\end{tabular}

In another study, researchers investigated the input vibration levels experienced during the initial and final stage of package distribution. In this study, Lansmont data recorders were directly mounted on the sidewalls of the cargo holds of two types of delivery vans and one type light delivery truck. These vehicles pick packages up from the sender and deliver them to the final recipient. To collect 
this data, the mounted data recorders were turned on each morning before the vehicles left for daily operations and their movement was monitored throughout the entire day. By the end of the study, over 50 hours movement was recorded near San Luis Obispo, CA [20].

During the analysis of the collected data, researchers developed a PDS breakpoint curve of the vertical vibration and determined the overall $\mathrm{G}_{\mathrm{rms}}$ value for each vehicle and axis of vibration. Although the vibration levels found in the $X$ and $Y$ axes were collected, they were found to be significantly lower than the vibration in the vertical $Z$ axis. All of the overall $G_{r m s}$ levels found are summarized below in table 8 .

Table 8: Overall vibration $\mathrm{G}_{\mathrm{rms}}$ levels observed in delivery vehicles [20]

\begin{tabular}{|c|c|c|c|}
\hline & Ford Van & Dodge Van & Freightliner Truck \\
\hline Longitudinal (x-axis) & 0.054 & 0.053 & 0.066 \\
\hline Lateral (y-axis) & 0.121 & 0.252 & 0.120 \\
\hline Vertical (z-axis) & 0.207 & 0.231 & 0.377 \\
\hline
\end{tabular}

In a another study, the vibration experienced while packages are moved on different types material handling equipment used in warehouses and at distribution hubs was investigated. The type of equipment that could be encountered in this segment of transport could be described in the following categories [21]. 
1.Transportation equipment used to move goods a short distance between locations.

E.g. carts, pallet jacks, converters, industrial jacks

2. Positioning equipment used to position goods in order to allow additional movement.

E.g. Hoists, dock levelers

3. Unit load formation equipment- used to consolidate packages to allow easier movement.

E.g. Pallets, totes, intermodal containers, stretch wrap,

4. Storage equipment- used for holding packages.

E.g. Racks, mezzanines, carousels

5.Identification and control equipment- used to coordinate proper material flow

E.g. Barcode scanners, RFID equipment

As many forms of material handling equipment do not have any suspension and hard caster wheels underneath them, damaging vibration levels can be easily incurred. Therefore, the findings from this study are valuable for the creation of accurate testing profiles that encompass the complete path of distribution.

After an analysis of the data collected in this study, a composite PDS plot was developed representing the vibration levels experienced. The breakpoints of this composite spectrum are included in table 9 below. From this data, it can be identified vibration of much higher frequency $(4-20 \mathrm{~Hz})$ is encountered during movement on the material handling equipment when compared to the vibration experienced from truck transport $(2-8 \mathrm{~Hz})$. The breakpoints from this newly developed PDS curve can be input into a vibration table and included in package performance testing. 
Table 9: Composite PDS breakpoints of material handling equipment [21]

\begin{tabular}{|c|c|}
\hline Frequency & $\begin{array}{c}\text { Power Density } \\
\left(\mathbf{G}^{2} / \mathbf{H z}\right)\end{array}$ \\
\hline $\mathbf{2}$ & 0.001 \\
\hline $\mathbf{5}$ & 0.01 \\
\hline $\mathbf{1 5}$ & 0.1 \\
\hline $\mathbf{2 5}$ & 0.1 \\
\hline $\mathbf{3 0}$ & 0.01 \\
\hline $\mathbf{1 0 0}$ & 0.001 \\
\hline
\end{tabular}

Just as the vibration experienced during ground transport has been studied and analyzed, the vibration present in aircraft transport has also been investigated in numerous past studies.

In a study completed by researchers at Amgen Inc, the vibration conditions experienced within their aircraft distribution routes were investigated. In order to collect this vibration data, four Lansmont SAVER 3x90 data recorders were directly mounted to the LD3 ULD shipping containers used within their transport. As these containers are directly fastened to the aircraft, the collected vibration data is closely representative of the true input vibration levels present [22]. 


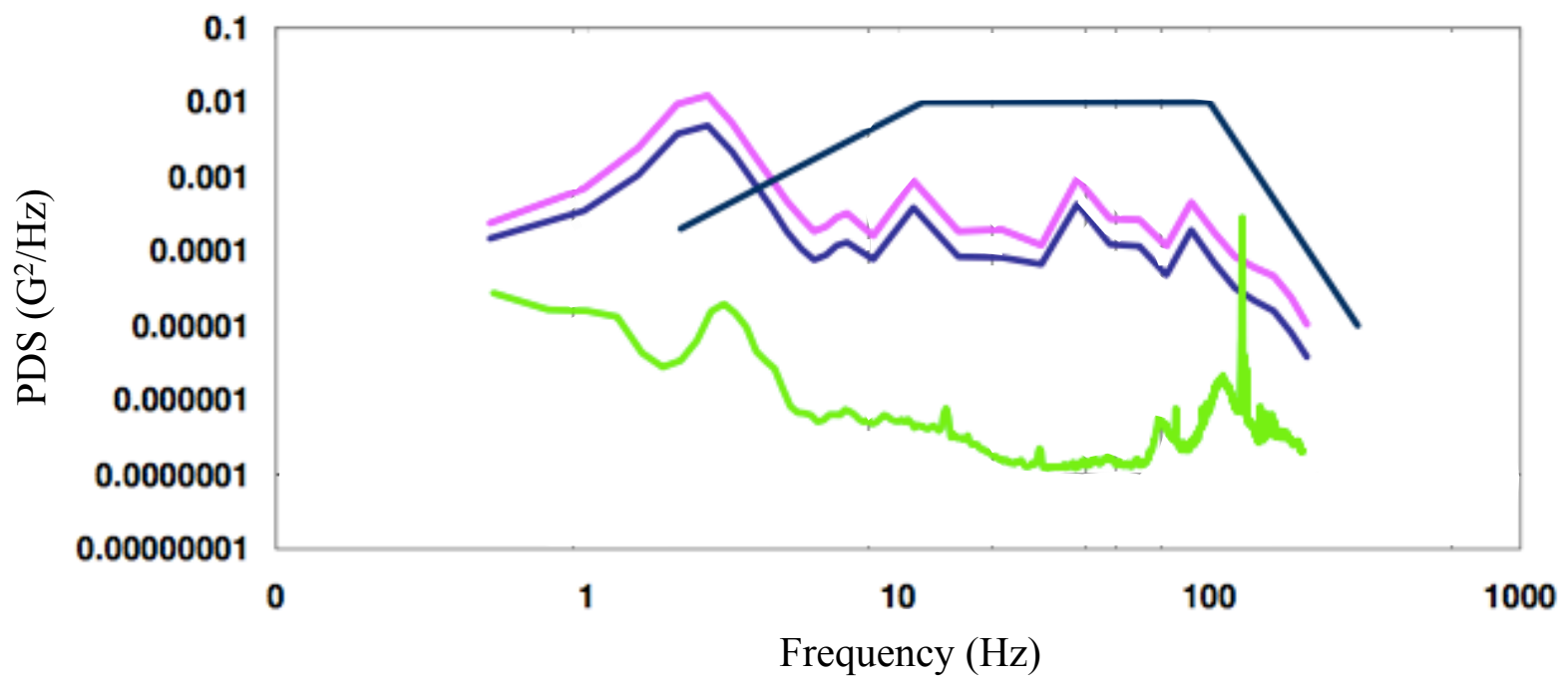

— Amgen Low Intensity Random Vibration (0.145 Grms)

- Amgen High Intensity Random Vibration $\left(0.222 \mathrm{G}_{\mathrm{rms}}\right)$

- Amgen Air Transport Composite Vibration ( $0.017 \mathrm{G}$ rms)

- ASTM Air Vibration Profile

Figure 9: Amgen distribution PDS curves before analysis [22]

After the collection of data, analysis was performed to create a PDS test profile representative of the upper $20 \%$ and lower $80 \%$ intensity of the input vibration present. A PDS plot of the collected raw data, before analysis was performed, is shown in figure 9.

The initial step of analysis was to determine the source of the large power intensity spike at the frequency of $125 \mathrm{~Hz}$. Through the performance of a resonance search test on the LD3 shipping container, this frequency was found to be a resonant frequency for this type of container. For this reason, the spike of 
vibration intensity was removed from the data analysis as it was not considered to directly represent input vibration. Rather, it was sourced from the ULD dollies responding to, and amplifying, the input vibration.

After narrowing the data being analyzed to only include input vibration, the atmospheric pressure data recorders were referenced to determine the times the plane was in flight. Once identified, the data could be filtered to only include vibration experienced during air transport as this portion of distribution was the primary focus of this study.

After identifying the vibration experienced during air transport, it was further analyzed to create two vibration test profiles. One profile representing the upper $20 \%$ and one for the lower $80 \%$ of vibration intensity data. The intensity level breakpoint was identified as $0.0305 \mathrm{G}_{\mathrm{rms}}$. Therefore, in order to create the Amgen High Intensity Airplane Random Vibration Profile, the average of all the data with intensities above this level were compiled. In similar terms, the data below this intensity level was averaged to create the Amgen Low Intensity Airplane Random Vibration Profile. The PDS plot with these profiles included is shown in figure 10 below. 


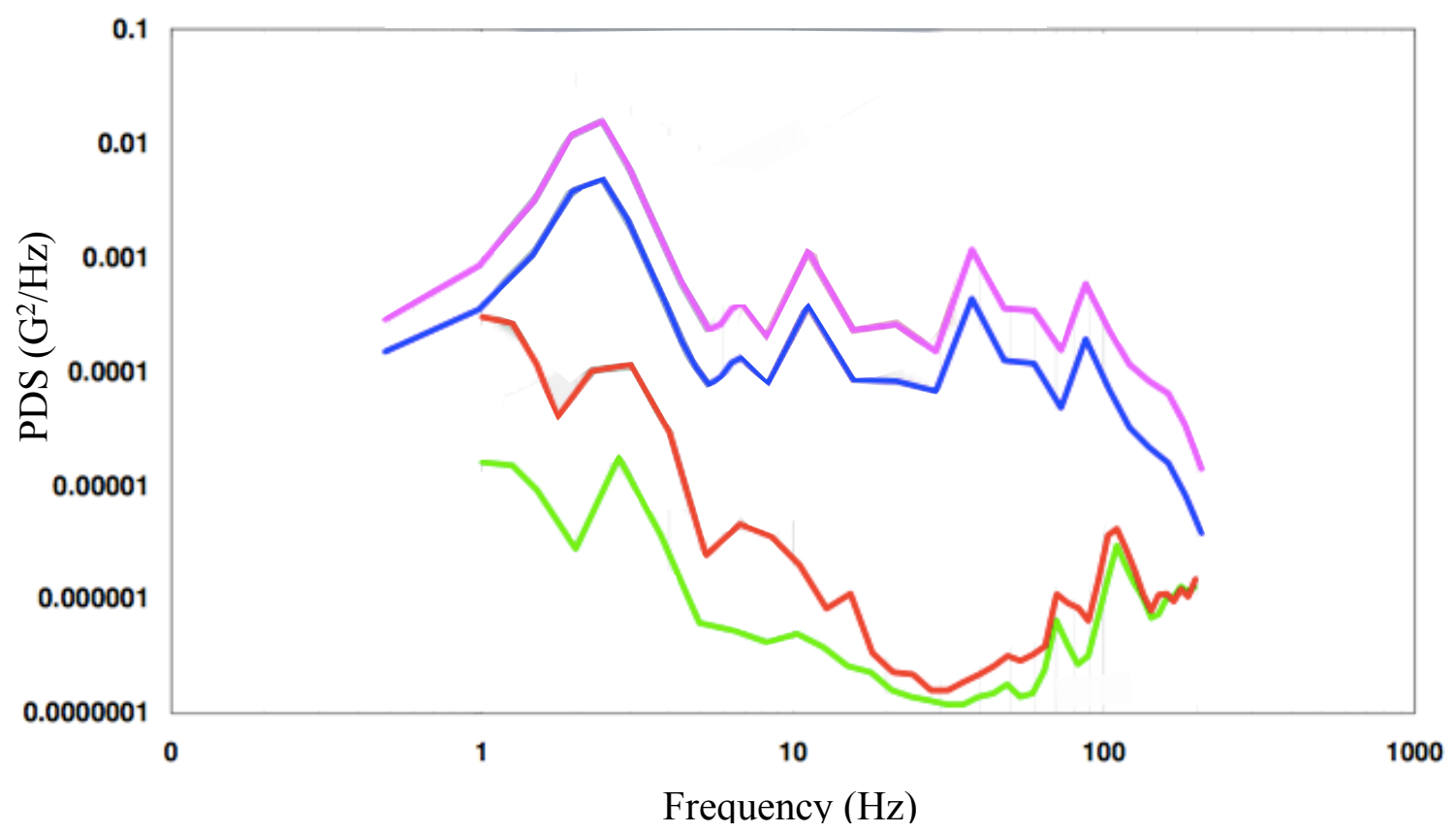

- Amgen Low Intensity Random Vibration $\left(0.145 \mathrm{G}_{\mathrm{rms}}\right)$

- Amgen High Intensity Random Vibration ( $\left.0.252 \mathrm{G}_{\mathrm{rms}}\right)$

- Amgen Low Intensity Airplane Random Vibration (0.017 Grms)

- Amgen High Intensity Airplane Random Vibration ( $\left.0.017 \mathrm{G}_{\mathrm{rms}}\right)$

Figure 10: Amgen distribution PDS curves after analysis [22]

In a different study, completed by researchers at Clemson State University, the vibration levels that could be experienced during transport on a twin turbo propeller feeder aircraft were investigated. The gathering of this data allowed for a better quantification of the vibration conditions experienced while packages are being transported in the next day or second day air package delivery service commonly used for modern distribution [23]. 
To complete this study, a Lansmont SAVER 9X30 data recorder was rigidly mounted directly to the sidewall of the Rockwell Turbocommander Twin Engine 690B AC90 aircraft cargo hold. This direct placement of the data recorder on the structure of the plane allowed for the accurate collection of valuable input vibration data. The recorders were set up to collect vibration data for a period of 2.048 seconds after a reoccurring 30 second timer triggered interval and whenever a signal trigger was enacted through experiencing acceleration levels of above $0.50 \mathrm{~g}$. The recorders were mounted and vibration data was collected on over 30 domestic flights ranging between 1 to 4 hours in duration. The direct details of data recorder setup is included in table 10.

Table 10: Data recorder setup used for analysis of feeder aircraft [23]

\begin{tabular}{|c|c|c|c|}
\hline \multicolumn{2}{|c|}{ Timer Triggered Data Parameter } & \multicolumn{2}{c|}{ Signal Triggered Data Parameter } \\
\hline Timer Trigger Wakeup Interval & Every 30 seconds & Trigger Threshold & $0.50 \mathrm{G}$ \\
\hline Sampling Rate & 1000 samples/sec & Signal Pre-trigger & $20 \%$ \\
\hline Record Time & 2.048 seconds & Sampling Rate & 1000 samples/sec \\
\hline Data Recording Mode & Fill/Stop & Record Time & 2.048 seconds \\
\hline \multirow{2}{*}{ Memory Allocation } & \multirow{2}{*}{$80 \%$} & Data Retention Mode & Max Overwrite \\
\cline { 3 - 4 } & & Memory Allocation & $20 \%$ \\
\hline
\end{tabular}

After data collection, the overall $\mathrm{G}_{\mathrm{rms}}$ level and peak acceleration level for each trigger type in each route of distribution was determined. A table outlining this vibration data is shown below in table 11. Where no signal trigger data is noted, no acceleration level greater than $0.05 \mathrm{G}$ was experienced. This analysis determined a cumulative $\mathrm{G}_{\mathrm{rms}}$ level of $0.062 \mathrm{G}$ was experienced for all timer triggered data, and $0.155 \mathrm{G}$ for signal triggered data. 
Table 11: Summary of feeder aircraft vibration [23]

\begin{tabular}{|c|c|c|c|c|}
\hline \multicolumn{2}{|c|}{ Flight Routes } & \multicolumn{2}{|c|}{ Overall $\mathrm{G}_{\mathrm{rms}}$} & \multirow{2}{*}{$\begin{array}{c}\text { Maximum } \\
\text { Acceleration (g) }\end{array}$} \\
\hline Origin & Destination & Timer Triggered & Signal & \\
\hline Oconee, SC & Columbia, SC & 0.065 & 0.170 & 1.13 \\
\hline Columbia, SC & Oconee, SC & 0.050 & No Data* & 0.32 \\
\hline Oconee, SC & Charleston, SC & 0.054 & 0.190 & 1.27 \\
\hline Charleston, SC & Oconee, SC & 0.054 & 0.119 & -0.63 \\
\hline Oconee, SC & Saluda, SC & 0.047 & No Data* & -0.33 \\
\hline Saluda, SC & Charleston, SC & 0.068 & 0.184 & 0.86 \\
\hline Charleston, SC & Oconee, SC & 0.058 & 0.173 & 0.74 \\
\hline Oconee, SC & Memphis, TN & 0.063 & 0.139 & 0.82 \\
\hline Memphis, TN & Oconee, SC & 0.059 & No Data* & 0.31 \\
\hline Oconee, SC & New York, NY & 0.060 & 0.153 & -1.48 \\
\hline New York, NY & Oconee, SC & 0.068 & 0.168 & -0.77 \\
\hline Oconee, SC & Knoxville, TN & 0.079 & 0.192 & -2.11 \\
\hline Knoxville, TN & Charleston, SC & 0.082 & 0.166 & 0.93 \\
\hline Charleston, SC & Columbia, SC & 0.067 & 0.173 & -0.67 \\
\hline Columbia, SC & Oconee, SC & 0.060 & 0.166 & 0.98 \\
\hline Oconee, SC & Jacksonville, FL & 0.088 & 0.174 & -0.61 \\
\hline Jacksonville, FL & Atlanta, GA & 0.063 & 0.161 & -0.94 \\
\hline Atlanta, GA & Oconee, SC & 0.079 & 0.156 & 1.38 \\
\hline Oconee, SC & Atlanta, GA & 0.070 & 0.156 & -0.91 \\
\hline Atlanta, GA & Oconee, SC & 0.061 & 0.153 & 0.74 \\
\hline Oconee, SC & Saluda, SC & 0.054 & No Data* & 0.47 \\
\hline Saluda, SC & Oconee, SC & 0.049 & 0.111 & 0.51 \\
\hline Oconee, SC & Atlanta, GA & 0.060 & 0.116 & 0.73 \\
\hline Oconee, SC & Charleston, SC & 0.053 & No Data* & -0.24 \\
\hline Charleston, SC & Oconee, SC & 0.052 & No Data* & -0.41 \\
\hline Oconee, SC & Atlanta, GA & 0.086 & 0.169 & 0.79 \\
\hline Oconee, SC & Columbia, SC & 0.054 & No Data* & -0.43 \\
\hline Columbia, SC & Oconee, SC & 0.046 & 0.167 & 0.81 \\
\hline Oconee, SC & Charleston, SC & 0.055 & 0.061 & 0.51 \\
\hline Charleston, SC & Oconee, SC & 0.057 & 0.156 & 0.68 \\
\hline \multirow{2}{*}{ Summary Data } & Average & 0.062 & 0.155 & $\mathrm{~N} / \mathrm{A}$ \\
\hline & Std. Dev. & 0.011 & 0.030 & N/A \\
\hline
\end{tabular}


Through the examination of figure 11 below, it is apparent the data collected after signal triggers was of greater intensity than the data collected after timer triggers. Although the overall shape is similar, the acceleration intensity level was greater for almost all the frequencies of movement.

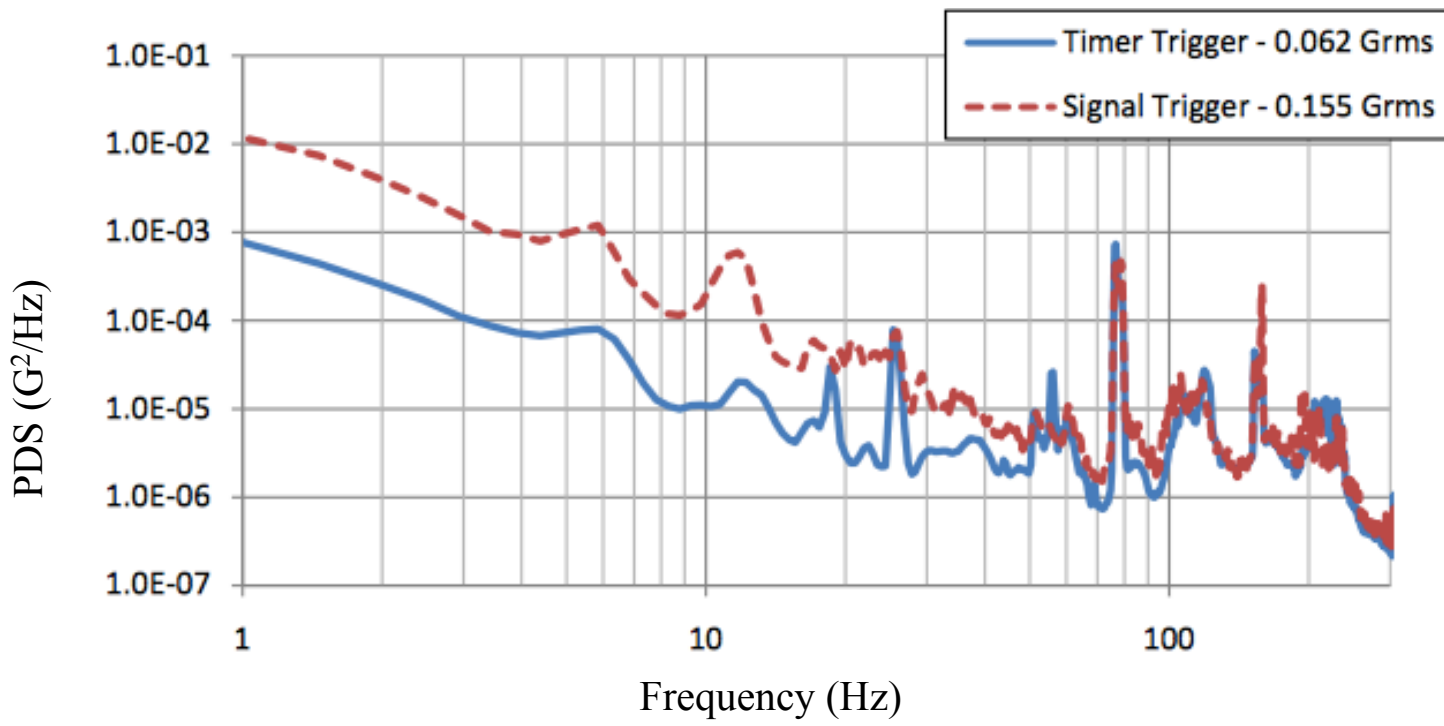

Figure 11: Cumulative average timer and signal triggered data collected[23]

When the vibration profiles observed in this study are compared to profiles found in past studies, several deviations are apparent. As can be seen in figure 12 below, a higher vibration intensity is present in both the ASTM D4169 and ISTA $4 \mathrm{AB}$ test specifications. However, in contrast, a lower overall vibration intensity is present in the study completed by Amgen where they investigated the vibration levels present in their distribution routes. Through the completion of a statistical analysis, the overall $\mathrm{G}_{\mathrm{rms}}$ level compiled from the timer and signal triggered data was determined to be statistically different from the profiles found in all three of these past studies. 


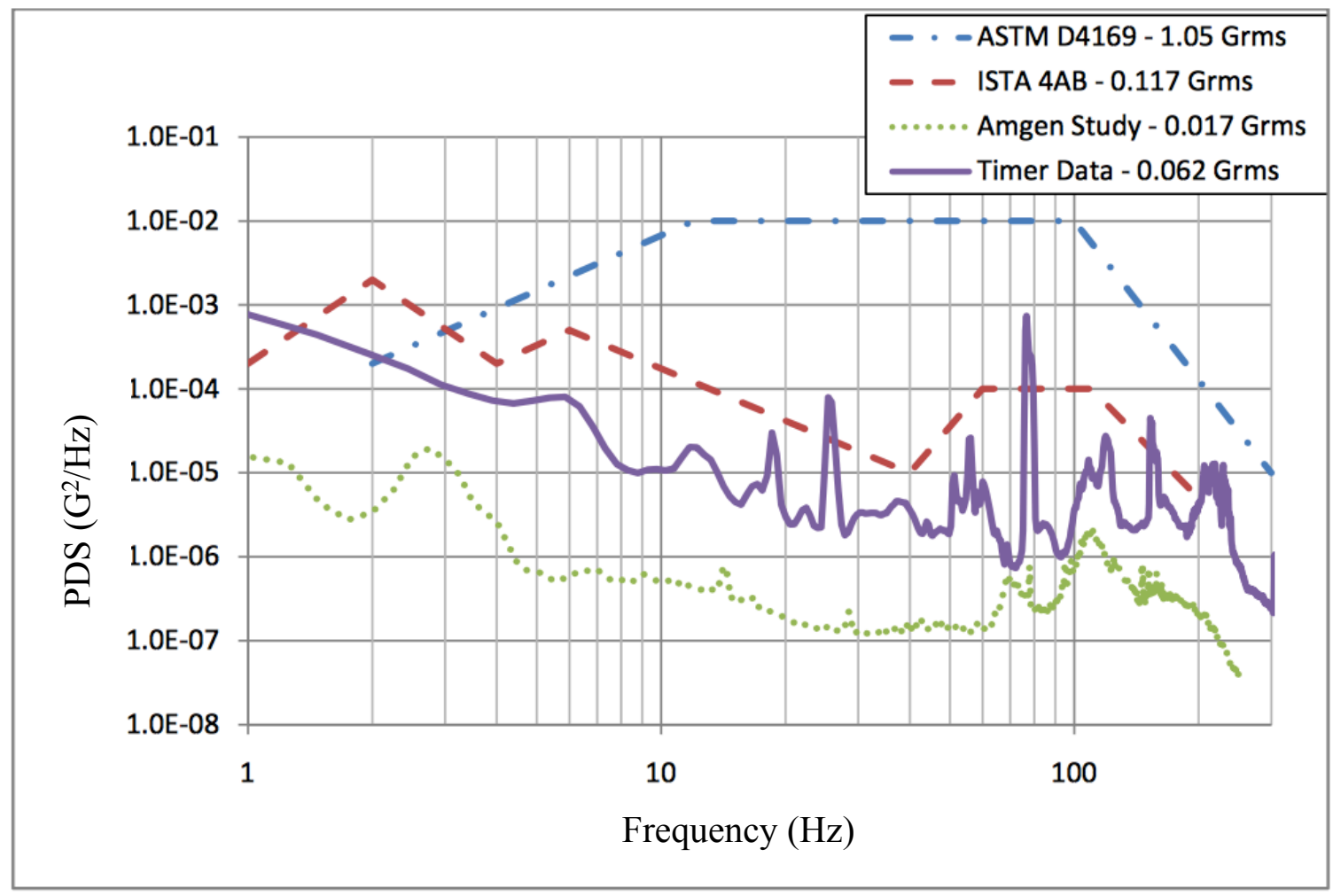

Figure 12: Comparative analysis of collected vibration data [23]

In another study completed by researchers at California Polytechnic State University, the effect of vibration occurring in low pressure conditions was investigated [24]. This study include a total of 32 different UN approved package types. To complete this study, the test packages were filled with water, sealed in accordance with the manufacturers torque specification, packed in secondary packaging as if they were being prepared for shipment, and conditioned for 24 hours to $73^{\circ} \mathrm{F}$ and $50 \%$ relative humidity. Once this conditioning was performed, the packages were placed in the most susceptible upside down and sideways package shipping orientations in a pressure chamber that was fastened to the top of the vibration table [24]. 
To conduct the test, the pressure within the chambers was reduced to $59.5 \mathrm{Kpa}$ (pressure equivalent of $14,000 \mathrm{ft})$ at a change rate of $305 \mathrm{~m}(1,000 \mathrm{ft})$ every 30-60 seconds. Once this low pressure level was achieved in the chamber, the vibration table was activated in order to induce vibration as specified in ASTM D 4169 assurance level II air and truck random vibration profile. This vibration test was run for a total of 30 minutes at these conditions. At the completion of 30 minutes, the pressure within the chamber was increased at a similar rate to which it was decreased at. When the pressure level had increased to sea level pressure, the samples were removed and inspected for any leaks that had been induced.

At the completion of this study, 15 of the 32 package types had begun leaking. In order to better characterize the effect different levels of pressure and vibration being experienced could have on package performance, additional tests were run with the level of pressure and vibration being altered.

The details of these test levels and the corresponding failure rates induced is shown below in table 12. All of the included vibration tests were run in accordance with ASTM D 4169 assurance level II air and truck random vibration. 
Table 12: Failure rates of varying levels of pressure and vibration [24]

\begin{tabular}{|c|c|}
\hline $\begin{array}{l}\text { Test Conditions } \\
\text { 1. Inclusion of ASTM D4169 Vibration Profile } \\
\text { 2. Included pressure level } \\
\text { 3. Duration of test }\end{array}$ & Package Failure Rate (\%) \\
\hline $\begin{array}{l}\text { 1. No vibration } \\
\text { 2. } 14,000 \mathrm{ft} \\
\text { 3. } 30 \mathrm{Mins}\end{array}$ & $\mathbf{0}$ \\
\hline $\begin{array}{l}\text { 1. Truck and Air vibration } \\
\text { 2. } 0 \mathrm{ft} \\
\text { 3. } 30 \mathrm{mins}\end{array}$ & 14 \\
\hline $\begin{array}{l}\text { 1. Truck only vibration } \\
\text { 2. } 8000 \mathrm{ft} \\
\text { 3. } 180 \mathrm{Min}\end{array}$ & 21 \\
\hline $\begin{array}{l}\text { 1. Truck and Air Vibration } \\
\text { 2. } 8000 \mathrm{ft} \\
\text { 3. } 180 \mathrm{~min}\end{array}$ & 29 \\
\hline $\begin{array}{l}\text { 1. Truck and Air Vibration } \\
\text { 2. } 14,000 \mathrm{ft} \\
\text { 3. } 30 \mathrm{mins}\end{array}$ & 50 \\
\hline
\end{tabular}

As can be seen from the findings above, there is a significant effect when vibration and low pressure are simultaneously experienced. For this reason, package performance testing should be completed to properly account for their effects.

\subsubsection{Shock}

Another damaging physical hazard commonly experienced throughout package distribution is varying levels of shock. As there is a possibility to incur shock whenever packages are in motion, it could be induced during the individual package movement of handling operations between different segments of 
transport, or while being included in a moving transport vehicle such as a truck or plane. The deceleration levels of a shock event could lead to many forms of material separation damage, such as tearing and cracking, or material deformation damage, such as crushing and creasing, to be induced on both the packaging parts or the contents within.

As shocks are commonly induced from the impact after a free fall drop, the severity of drops is identified through the free fall drop height. In the case where free fall is not the primary contributor of energy, the shock severity is identified as the equivalent drop height that would need to be incurred in order to receive an the same level of shock. To determine free fall drop severity in this manner, the height of the experienced free falls can be automatically measured with data recorders through the use of the "real drop height" calculation method. This method includes measuring the time the package spends in the zero G state (free fall) that occurs between a single g state (motionless) and a state of several g's (impact shock). The "real drop height" can be quantified for each particular drop event with the following equation [25].

$$
h=1 / 2 g t^{2}
$$

$h=$ free fall drop height, $m$ or in.

$g=$ acceleration due to gravity, $9.81 \mathrm{~m} / \mathrm{s}^{2}$ or $386.4 \mathrm{in} / \mathrm{s}^{2}$

$t=$ free fall duration, expressed in seconds

Another method used to determine shock severity includes analyzing the shock pulse waveform recorded by the triaxial accelerometer within the data recorder. 
This method, known as the "effective drop height" calculation method, analyzes the amount of velocity change present in the $\mathrm{X}, \mathrm{Y}$, and $\mathrm{Z}$ axis pulse waveforms and the resultant velocity change is calculated with the following equation [26].

$$
\Delta v r=\left(\Delta v x^{2}+\Delta v y^{2}+\Delta v z^{2}\right) 0.5
$$

The "real drop height" and "effective drop height" calculation methods for determining shock severity have been used in many previous studies. After a review of some of these studies, a few key findings were identified. When the equivalent drop heights found with these two methods were very similar (within $10 \%)$, the height determined with the "zero-g channel" method was identified as having greater accuracy. Also, when the height determined with the "effective drop height" method was much lower than the height determined with the "real drop height" calculation method, these events were classified as "tosses". In a case like this, the total experienced shock force is much lower than would be incurred if the package was only traveling vertically while in a zero $g$ state of free fall.

As the damaging effects can easily be identified in both packaging and products within, there has been a significant amount of research completed to identify the levels of shock experienced in domestic and international shipping environments. This has allowed accurate pre-shipment performance testing standards to be developed for use to help further improve package designs.

In one study, the domestic shipping environment between East Lansing, MI and San Luis Obispo, CA was analyzed to investigate the drops experienced while 
being transported within four different shipment carriers- DHL, Fedex, UPS, and USPS [26, 21]. Although the highest drop seen during all these shipments was $1.87 \mathrm{~m}$, the drop height occurrence level for $95 \%$ of the packages ranged from a height of 0.68 to $0.86 \mathrm{~m}$ in the Next Day air shipping service and from 0.66 to $1.16 \mathrm{~m}$ in Second Day air shipping service. A table with occurrence level drop heights of $90 \%, 95 \%$, and $99 \%$ of package drops is shown below in table 13 .

Table 13: E. Lansing, Ml / San Luis Obispo, CA distribution shock data [27]

\begin{tabular}{|c|c|c|c|c|c|c|c|c|}
\hline & \multicolumn{4}{|c|}{ Next-day or Express } & \multicolumn{4}{c|}{ Second-day or Priority } \\
\cline { 2 - 10 } & DHL & FedEx & UPS & USPS & DHL & FedEx & UPS & USPS \\
\hline Number of drops & 184 & 128 & 117 & 156 & 168 & 182 & 104 & 66 \\
\hline $\begin{array}{c}\text { Maximum drop height } \\
\text { (m) }\end{array}$ & 1.45 & 1.77 & 1.23 & 1.43 & 1.01 & 0.89 & 1.63 & 1.87 \\
\hline $\begin{array}{c}\text { Drop height at 99\% } \\
\text { occurrence (m) }\end{array}$ & 1.14 & 1.04 & 1.04 & 1.42 & 0.96 & 0.88 & 1.02 & 1.86 \\
\hline $\begin{array}{c}\text { Drop height at 95\% } \\
\text { occurrence (m) }\end{array}$ & 0.86 & 0.7 & 0.68 & 0.76 & 0.76 & 0.66 & 0.76 & 1.16 \\
\hline $\begin{array}{c}\text { Drop height at 90\% } \\
\text { occurrence (m) }\end{array}$ & 0.62 & 0.52 & 0.46 & 0.56 & 0.56 & 0.44 & 0.48 & 0.88 \\
\hline
\end{tabular}

Furthermore, this study also investigated how packages were oriented during impacts and found a majority of the impacts were sustained on the package faces. The full proportion breakdown of package orientations during impacts is presented in table 14 . 
Table 14: Impact orientation proportions [27]

\begin{tabular}{|c|c|c|c|c|}
\hline \multirow{2}{*}{} & \multicolumn{3}{|c|}{ Proportion of Impact Orientation (\%) } \\
\cline { 3 - 5 } & Face & Edge & Corner \\
\hline \multirow{3}{*}{ Next-day } & FHL & 83.69 & 7.61 & 8.69 \\
\cline { 2 - 5 } & Fedex & 91.41 & 6.25 & 2.34 \\
\cline { 2 - 5 } & UPS & 93.16 & 2.56 & 4.27 \\
\hline \multirow{3}{*}{$\begin{array}{c}\text { Second- } \\
\text { day }\end{array}$} & DHL & 82.17 & 7.75 & 10.08 \\
\cline { 2 - 5 } & Fedex & 88.46 & 6.04 & 5.49 \\
\cline { 2 - 5 } & UPS & 93.27 & 4.81 & 1.92 \\
\hline
\end{tabular}

In a different study conducted by researchers at California Polytechnic State University and Michigan State University, the distribution from East Lansing, MI to San Francisco, CA, as well as from East Lansing, MI to Orlando, FL, was investigated [28]. This study focused on the effect that package size and weight has on the height of drops experienced. In addition, the effect of warning labels placed on the exterior of the package in reducing drop severity was also investigated. A total of five different package sizes and weights were included in this study and are presented in table 15 below [28].

Table 15: Test package weights and dimensions [28]

\begin{tabular}{|c|c|c|}
\hline Package \# & Dimensions (In) & Weight (lbs) \\
\hline $\mathbf{1}$ & $0.18 \times 0.19 \times 0.13 \mathrm{~m}$ & 1.9 \\
\hline $\mathbf{2}$ & $0.21 \times 0.21 \times 0.16 \mathrm{~m}$ & 2.2 \\
\hline $\mathbf{3}$ & $0.26 \times 0.27 \times 0.21 \mathrm{~m}$ & 3.7 \\
\hline $\mathbf{4}$ & $0.31 \times 0.32 \times 0.26 \mathrm{~m}$ & 4.8 \\
\hline $\mathbf{5}$ & $0.36 \times 0.37 \times 0.31 \mathrm{~m}$ & 5.5 \\
\hline
\end{tabular}


After collecting data, a few conclusions were identified. First, exact package size and weight had no significant effect on the incurred drop heights for packages classified as small and light. Second, the addition of fragility warning labels placed on the exterior of the packages did not have a significant effect on reducing the severity of experienced shocks. Lastly, after an investigation of how the packages were oriented during drops, it was found that for every time a package was dropped on a corner, it was likely to experience two drops on an edge and 3-5 drops on a face. This proportion of impact orientations suggests a majority of impacts were incurred during travel through automatic handling equipment rather than during manual handling operations. A total of $95 \%$ of all the drops occurred from a height ranging from 0.86 to $1.447 \mathrm{~m}$, depending on the size and weight of the package. A summary of all the results found in this study are presented in table 16 below.

Table 16: Collected domestic drop data [28]

\begin{tabular}{|c|c|c|c|c|c|}
\hline & Package 1 & Package 2 & Package 3 & Package 4 & Package 5 \\
\cline { 2 - 6 } & \multicolumn{2}{|c|}{ Smallest / Lightest } & \multicolumn{2}{c|}{ Largest / Heaviest } \\
\hline Number of shocks & 172 & 163 & 176 & 193 & 193 \\
\hline $\begin{array}{c}\text { Maximum drop } \\
\text { height (m) }\end{array}$ & 1.393 & 1.541 & 1.001 & 1.201 & 1.851 \\
\hline $\begin{array}{c}\text { Drop height at } \\
\text { 99\% occurrence } \\
\text { (m) }\end{array}$ & 1.295 & 1.447 & 0.939 & 1.016 & 0.863 \\
\hline $\begin{array}{c}\text { Drop height at } \\
\text { 95\% occurrence } \\
\text { (m) }\end{array}$ & 0.838 & 0.762 & 0.635 & 0.635 & 0.609 \\
\hline $\begin{array}{c}\text { Drop height at } \\
\text { 90\% occurrence } \\
\text { (m) }\end{array}$ & 0.558 & 0.609 & 0.406 & 0.457 & 0.457 \\
\hline
\end{tabular}


Just as the shocks experienced in the domestic shipping environment have been analyzed, a study completed by researchers at Michigan State University quantified the occurrence of shocks in the international shipping environment. In this study, 12 midsize $(0.36 \mathrm{~m} \times 0.34 \mathrm{~m} \times 0.34 \mathrm{~m})$ and lightweight $(6.5 \mathrm{~kg})$ packages, containing an IST Environmental Data Recorder, were sent from East Lansing, MI to Valencia, Spain- six through DHL shipping service and six through Fedex shipping service. In addition, the effect of a fragility warning label placed on the exterior of the package to reduce the severity of shocks incurred was also investigated through the placement of labels like this on half of the test packages. After the collection of data, analysis was performed and the drop height for $90 \%$, $95 \%$, and $99 \%$ occurrence was identified. Additionally, as can be found in the table presented below, a significant effect of a warning label being placed on the exterior of the package is only apparent during drops seen at the $99 \%$ occurrence level in DHL shipments [26].

Table 17: Collected international drop data [26]

\begin{tabular}{|c|c|c|c|c|}
\hline & \multicolumn{2}{|c|}{ Fedex } & \multicolumn{2}{c|}{ DHL } \\
\cline { 2 - 5 } & No Label & Label & No Label & Label \\
\hline Ave. number of drops & 21 & 24 & 36 & 29 \\
\hline Maximum drop height (m) & 1.24 & 1.21 & 1.11 & 0.63 \\
\hline $\begin{array}{c}\text { 99\% occurrence level } \\
\text { drop height (m) }\end{array}$ & 1.06 & 0.98 & 0.89 & 0.58 \\
\hline $\begin{array}{c}\text { 95\% occurrence level } \\
\text { drop height (m) }\end{array}$ & 0.63 & 0.59 & 0.52 & 0.52 \\
\hline $\begin{array}{c}\text { 90\% occurrence level } \\
\text { drop height (m) }\end{array}$ & 0.39 & 0.37 & 0.38 & 0.42 \\
\hline Mean drop height & 0.21 & 0.20 & 0.21 & 0.20 \\
\hline
\end{tabular}


In addition to studies being completed to identify the height of drops experienced, one study investigated the proportion of how packages were moving immediately before incurring impact. Specifically, this study aimed to identify if a "drop" (vertical displacement), a "toss" (both vertical and lateral displacement), or a "kick" (lateral displacement) was occurring immediately before the impact shock. Additionally, this study also investigated the effect of the package size and weight on the occurrence and severity of the experienced shocks.

To complete this study, 32 test packages of three different sizes and weights (table 18) were sent in round trip shipments through the UPS distribution system. Five packages were included for each configuration and the duration of each shipment was 10 days.

Table 18: Test package weight and dimensions [29]

\begin{tabular}{|c|c|c|c|}
\hline \multicolumn{2}{|c|}{ Dimensions (in) } & \multicolumn{2}{c|}{ Weight (lbs) } \\
\hline Small & $12 \times 12 \times 12$ & Light & 20 \\
\hline Medium & $18 \times 18 \times 16$ & Medium & 30 \\
\hline Large & $26 \times 20 \times 19$ & Heavy & 45 \\
\hline
\end{tabular}

At the conclusion of the study, it was found the most common shock type incurred among all package configuration was a "kick". As this was a lateral only impact, it is reasonable to conclude these impacts were from the automatic handling machines present at distribution hubs and sortation facilities. Furthermore, almost all small and medium sized package configurations incurred more "tosses" than "drops". This leads to the conclusion packages of these sizes 
are more often manually handled. Lastly, as large packages experienced a greater amount of "drops" than "tosses", it is reasonable to conclude packages of this size do not receive much manual handling.

Table 19: Proportion of impact type occurrence (\%) [29]

\begin{tabular}{|c|c|c|c|}
\hline $\begin{array}{c}\text { Test Package Configuration } \\
\text { (Size / Weight) }\end{array}$ & "Kicks" & "Tosses" & "Drops" \\
\hline Small / Medium & $46 \%$ & $33 \%$ & $21 \%$ \\
\hline Small / Light & $47 \%$ & $28 \%$ & $31 \%$ \\
\hline Medium / Heavy & $37 \%$ & $33 \%$ & $30 \%$ \\
\hline Medium / Medium & $45 \%$ & $33 \%$ & $22 \%$ \\
\hline Medium / Light & $43 \%$ & $34 \%$ & $23 \%$ \\
\hline Large / Heavy & $33 \%$ & $33 \%$ & $33 \%$ \\
\hline Large / Medium & $41 \%$ & $25 \%$ & $34 \%$ \\
\hline
\end{tabular}

Lastly, it was concluded package size had no significant effect on the experienced drop height and package weight only had a significant effect on small size packages. A summary of the drop data for each size and weight package configuration can be found in table 20,21 , and 22 below. 
Table 20: Summary of drop data for small packages [29]

\begin{tabular}{|c|c|c|c|c|c|c|}
\hline Size & Weight & $\begin{array}{c}\text { Test Package } \\
\text { Number }\end{array}$ & $\begin{array}{c}\text { Total Number } \\
\text { of Drops }\end{array}$ & $\begin{array}{l}\text { Max Height } \\
\text { (in) }\end{array}$ & $\begin{array}{l}\text { Min Height } \\
\text { (in) }\end{array}$ & $\begin{array}{l}\text { Ave Height } \\
\text { (in) }\end{array}$ \\
\hline \multirow{10}{*}{ Small } & \multirow{5}{*}{ Light } & 1 & 9 & 32.3 & 0.3 & 9.7 \\
\hline & & 2 & 7 & 14.2 & 1.1 & 6.3 \\
\hline & & 3 & 8 & 14.3 & 0.3 & 6.4 \\
\hline & & 4 & 11 & 42.1 & 1.6 & 12.4 \\
\hline & & 5 & 2 & 2.8 & 0.5 & 1.6 \\
\hline & \multirow{5}{*}{ Medium } & 1 & 2 & 23 & 2.8 & 12.1 \\
\hline & & 2 & 1 & 14.7 & - & 14.7 \\
\hline & & 3 & 10 & 23.1 & 1.2 & 15.4 \\
\hline & & 4 & 1 & 8.7 & - & 8.7 \\
\hline & & 5 & 4 & 9.9 & 2.6 & 4.9 \\
\hline \multicolumn{3}{|c|}{ Small Light Average } & 7.4 & 21.1 & 0.8 & 7.3 \\
\hline \multicolumn{3}{|c|}{ Small Medium Average } & 3.6 & 17.9 & 2.2 & 11.2 \\
\hline \multicolumn{3}{|c|}{ Total Small Average } & 5.5 & 19.5 & 1.3 & 9.2 \\
\hline
\end{tabular}


Table 21: Summary of drop data for medium packages [29]

\begin{tabular}{|c|c|c|c|c|c|c|}
\hline Size & Weight & $\begin{array}{c}\text { Test Package } \\
\text { Number }\end{array}$ & $\begin{array}{c}\text { Total Number } \\
\text { of Drops }\end{array}$ & $\begin{array}{l}\text { Max Height } \\
\text { (in) }\end{array}$ & $\begin{array}{l}\text { Min Height } \\
\text { (in) }\end{array}$ & $\begin{array}{l}\text { Ave Height } \\
\text { (in) }\end{array}$ \\
\hline \multirow{15}{*}{ Medium } & \multirow[t]{5}{*}{ Light } & 1 & 4 & 13 & 0.2 & 4.8 \\
\hline & & 2 & 14 & 13.9 & 0.4 & 3.4 \\
\hline & & 3 & 13 & 15.7 & 0.3 & 4.7 \\
\hline & & 4 & 15 & 27.6 & 0.4 & 4.9 \\
\hline & & 5 & 17 & 37.2 & 0.4 & 8.8 \\
\hline & \multirow{5}{*}{ Medium } & 1 & 1 & 0.7 & - & 0.7 \\
\hline & & 2 & 12 & 13.2 & 0.2 & 4.5 \\
\hline & & 3 & 13 & 40.7 & 0.4 & 9.6 \\
\hline & & 4 & 0 & 0 & 0 & 0 \\
\hline & & 5 & 4 & 15.2 & 1 & 7.3 \\
\hline & \multirow{5}{*}{ Heavy } & 1 & 10 & 9.1 & 0.5 & 4.6 \\
\hline & & 2 & 5 & 30.9 & 0.7 & - \\
\hline & & 3 & 2 & 11.2 & 1.1 & 6.1 \\
\hline & & 4 & 5 & 2.2 & 0.5 & 1.4 \\
\hline & & 5 & 16 & 28.9 & 0.8 & 7.5 \\
\hline \multicolumn{3}{|c|}{ Medium Light Average } & 12.6 & 21.5 & 0.3 & 4.8 \\
\hline \multicolumn{3}{|c|}{ Medium Medium Average } & 6 & 14 & 0.4 & 4.4 \\
\hline \multicolumn{3}{|c|}{ Medium Heavy Average } & 7.6 & 16.5 & 0.7 & 4.9 \\
\hline \multicolumn{3}{|c|}{ Total Medium Average } & 8.7 & 17.3 & 0.5 & 4.9 \\
\hline
\end{tabular}


Table 22: Summary of drop data for large packages [29]

\begin{tabular}{|c|c|c|c|c|c|c|}
\hline Size & Weight & $\begin{array}{c}\text { Test Package } \\
\text { Number }\end{array}$ & $\begin{array}{c}\text { Total Number } \\
\text { of Drops }\end{array}$ & $\begin{array}{l}\text { Max Height } \\
\text { (in) }\end{array}$ & $\begin{array}{l}\text { Min Height } \\
\text { (in) }\end{array}$ & $\begin{array}{l}\text { Ave Height } \\
\text { (in) }\end{array}$ \\
\hline \multirow{10}{*}{ Large } & \multirow{5}{*}{ Medium } & 1 & 5 & 12.5 & 0.4 & - \\
\hline & & 2 & 15 & 19.8 & 0.5 & 7.7 \\
\hline & & 3 & 9 & 12.6 & 0.9 & 5 \\
\hline & & 4 & 10 & 30.1 & 1.5 & - \\
\hline & & 5 & 3 & 17.5 & 1.2 & 11.8 \\
\hline & \multirow{5}{*}{ Heavy } & 1 & 6 & 10.7 & 2.2 & 5.9 \\
\hline & & 2 & 3 & 18.8 & 1 & 8.7 \\
\hline & & 3 & 1 & 6.9 & - & 6.9 \\
\hline & & 4 & 5 & 17 & 0.9 & 6.2 \\
\hline & & 5 & 1 & 15 & - & - \\
\hline \multicolumn{3}{|c|}{ Large Medium Average } & 8.4 & 18.5 & 0.9 & 8.2 \\
\hline \multicolumn{3}{|c|}{ Large Heavy Average } & 3.2 & 13.7 & 1.4 & 6.9 \\
\hline \multicolumn{3}{|c|}{ Total Large Average } & 5.8 & 16.1 & 1.1 & 7.5 \\
\hline
\end{tabular}




\subsection{OBJECTIVES}

In order to better account for the "normal" hazard levels that could be experienced throughout the modern air parcel distribution environment, this study was completed with the following objectives.

1) Quantify the climatic hazard levels of temperature, relative humidity, and atmospheric pressure experienced in the international and domestic air parcel distribution environment.

2) Quantify the physical hazard levels of shock and vibration experienced in the international and domestic air parcel distribution environment.

3) Analyze the collected data on the climatic and physical hazard levels and create composite package performance testing profiles for each hazard element. These profiles will represent the normal conditions experienced throughout the entire air parcel distribution environment. 


\subsection{MATERIALS AND METHODS}

\subsection{Data Recording Test Package Construction}

In order to collect data on the physical and climatic hazard levels found in normal air parcel distribution, test packages were constructed and sent through actual distribution routes. These routes included transport from San Luis Obispo, CA to five domestic and four international locations. Once the test packages arrived at each of these locations, they were turned around and sent back to San Luis Obispo. This allowed for data collection in both directions of travel in all distribution routes.

Each of the test packages were constructed to record the physical and climatic hazard levels encountered throughout a complete route of distribution. As no single data recorder could be found to collect accurate severity level data for all of these conditions, two separate recorders were mounted within each test package- one to quantify the physical hazard levels, and another to capture the climatic hazard levels.

For the physical hazards of vibration and shock, the SAVER 3X90 data recorder was selected(Figure 13). This instrument, manufactured by Lansmont Corporation (Monterey, Ca), is widely used throughout the packaging industry for the analysis of such conditions. Before each shipment, the SAVER data recorders were initialized with the following settings applied. 
Minimum timer triggered sampling: 15 minutes

Trigger threshold level: $0.5 \mathrm{~g}$

Minimum sampling rate: 1000 samples per second

Minimum recording window: 1.024 seconds

Sample size: 1024

These settings triggered the recorders to collect data whenever a signal threshold level of $0.5 \mathrm{~g}$ was experienced and at a reoccurring 15 minute time interval. When either of these triggers were enacted, the SAVER data recorders collected 1024 corresponding frequency and power density breakpoints at a rate of 1000 samples per second.

To quantify the varying levels of climatic hazards, the SD700 data recorder, manufactured by Extech Instruments (Waltham, MA), was selected(Figure 13). These recorders were set to capture the temperature, humidity, and atmospheric pressure levels in 10 minute time intervals. Although this setting does not capture every instant throughout the entire path of distribution, it does present a good summary of the levels that could be experienced within all the different segments of transport. The SD700 data recorders were capable of identifying the following climatic hazard levels.

Atmospheric Pressure:

Range: $10 \mathrm{hPa}$ to $1,100 \mathrm{hPa}$

Resolution of $0.1 \mathrm{hPa}$

\section{Temperature}

Range: $0^{\circ} \mathrm{C}$ to $60^{\circ} \mathrm{C}$

Resolution of $0.1^{\circ} \mathrm{C} \pm 0.8^{\circ} \mathrm{C}$ 


\section{Relative Humidity}

Range: $10 \%$ to $90 \% \mathrm{RH}$

Within each test package, both of the data recorders were fastened to a fixture constructed out of 1"x1" extruded aluminum T-slot framing material, manufactured by $80 / 20$ Inc. Although the use of expanded polystyrene foam was considered as a fixture material, the aluminum T-slot framing was selected as it allowed a much lower level of shock and vibration force attenuation.

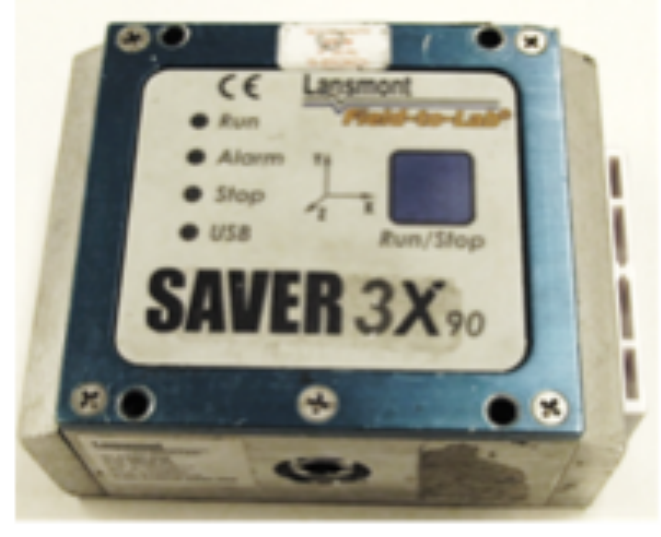

Lansmont SAVER 3X90

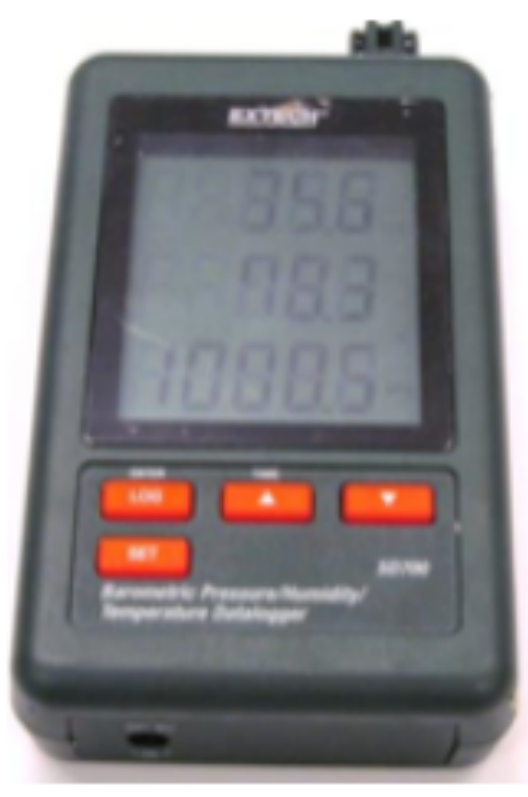

Extech SD700

Figure 13: Physical and climatic data recorders

In each test package, the physical data recorder was secured with fasteners to an aluminum plate mounted on the center crossbar of the fixture to allow it to be positioned in the geometric center of the test package. The climatic monitor was 
mounted on the opposite side of this center crossbar. To allow the climatic data recorder to accurately capture the environmental conditions present on the exterior of the test packages, four ventilation holes were cut in the upper flaps of the test package shippers.

As this study focused on conditions within "normal" package distribution, design aspects of the test packages were chosen to allowed them to be representative of a "normal" package. Specifically, the test package fixture was sized to snugly fit inside a regular slotted container made out of C-flute corrugated kraft fiberboard with external dimensions of $36.1 \mathrm{~cm} \mathrm{~L} \mathrm{x} 31.1 \mathrm{~cm} \mathrm{~W} \mathrm{x} 21.5 \mathrm{~cm} \mathrm{H}(141 / 4 "$ $\left.\times 121 / 4^{\prime \prime} \times 81 / 2 "\right)($ Figure 14). The shipper dimension, shipper box style, and shipper material were selected as they are common attributes of packages that are often shipped in normal air parcel distribution.

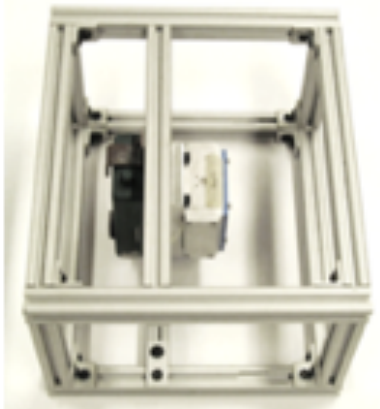

Data recorders mounted on aluminum fixture

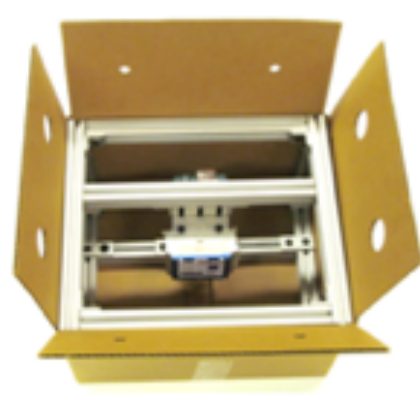

Aluminum fixture placed in RSC shipper

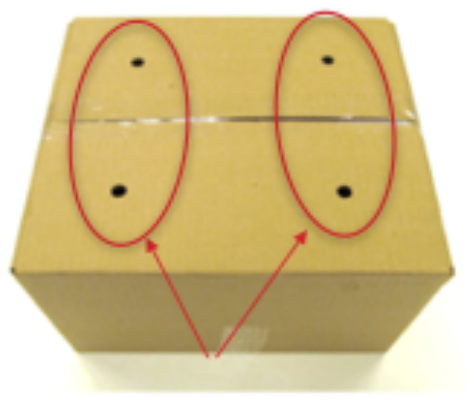

Sealed RSC shipper with ventilation holes

Figure 14: Test package assembly 


\subsection{Data Collection Routes}

In order to collect distribution hazard level data representative of levels experienced in "normal" air parcel distribution, three instrumented test packages were sent in round trip distribution to a total of five domestic and four international locations. These locations required travel through a wide variety of geographic regions in order to capture some of the varying hazard levels present in the infinitely variable distribution environment. All of these locations are identified below in table 23 and graphically represented in figure 15 and 16 .

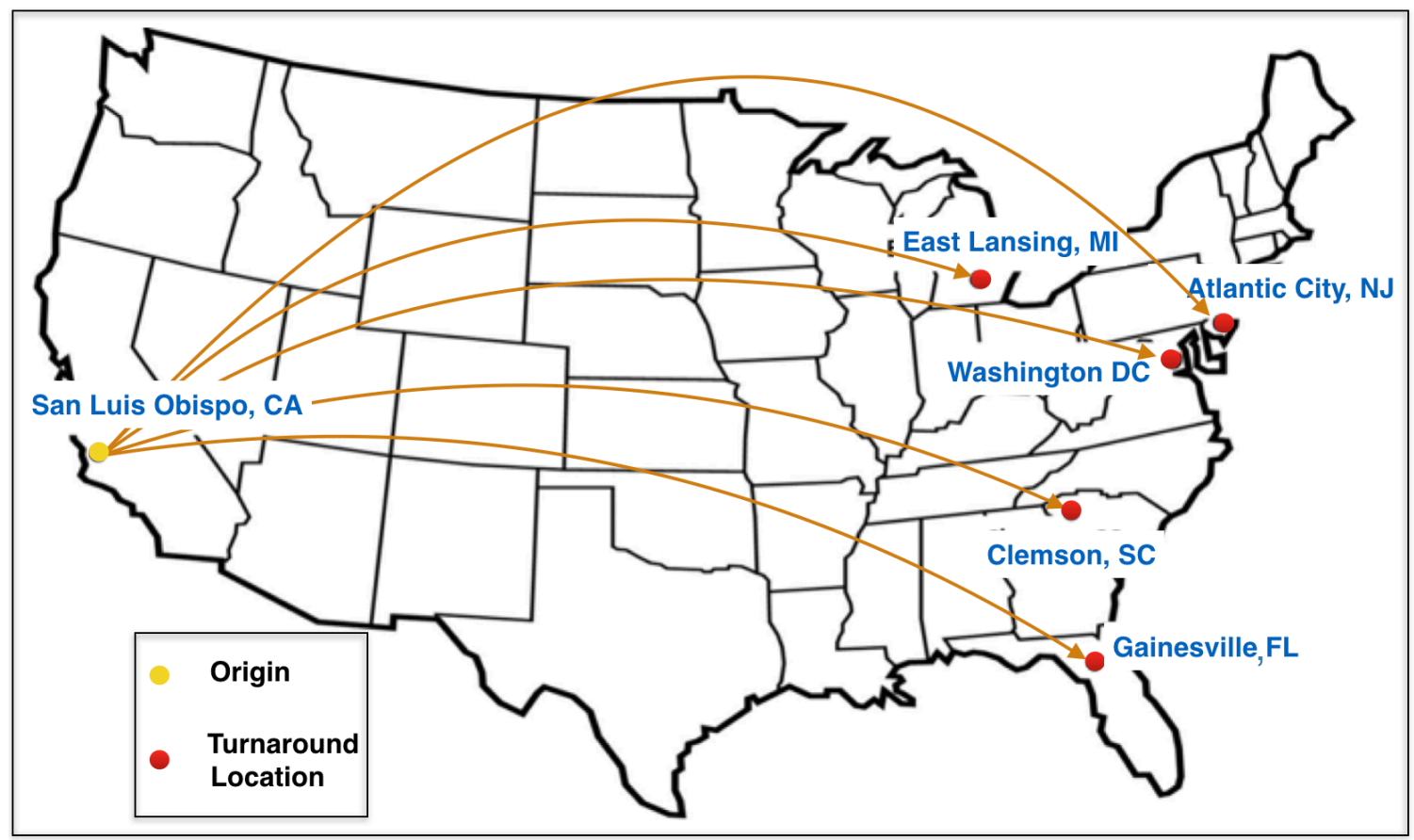

Figure 15: Domestic distribution routes investigated 


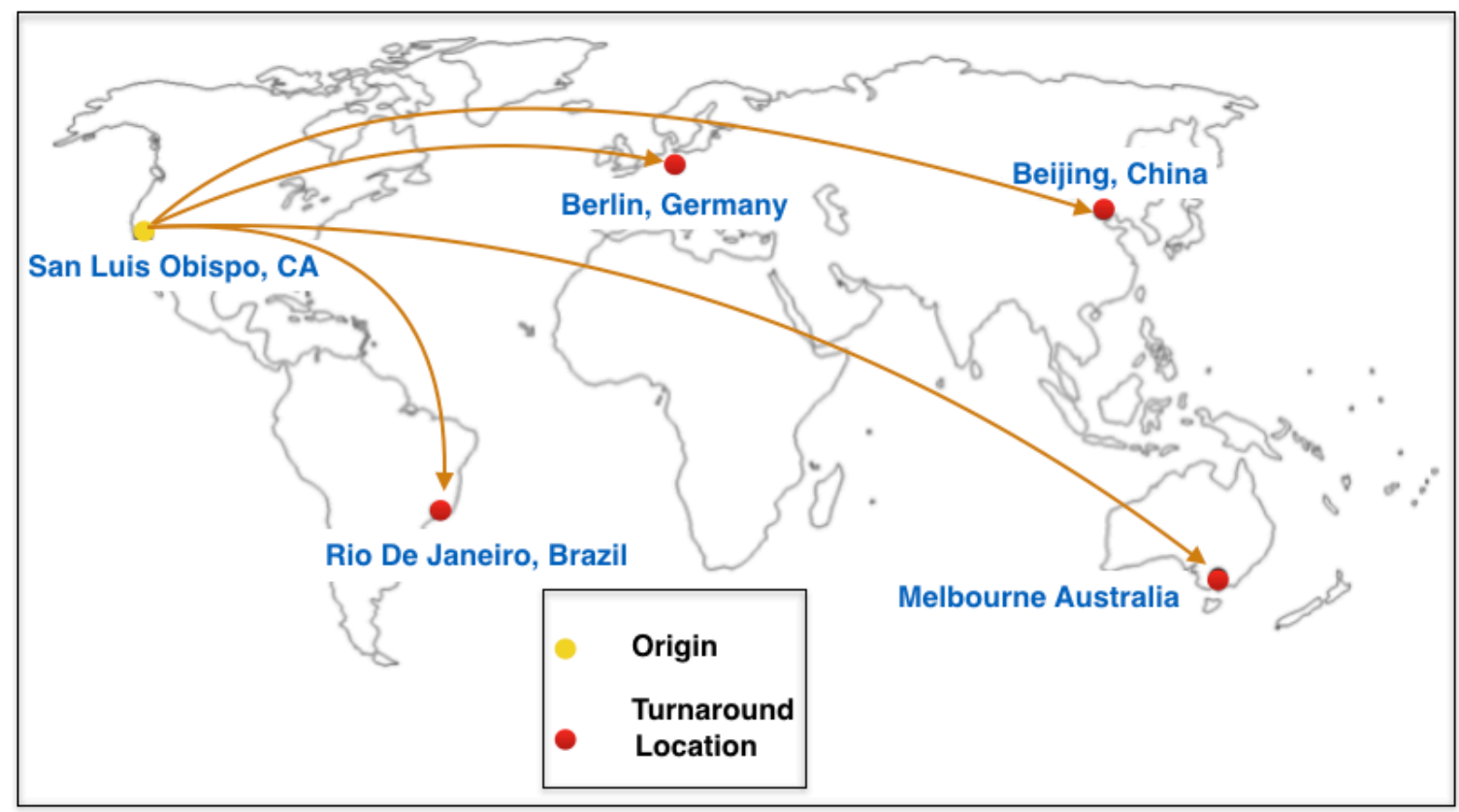

Figure 16: International distribution routes investigated

Table 23: Domestic and International distribution routes investigated

\begin{tabular}{|c|c|c|c|}
\hline Shipment & Origin Location & Turn Around Location & Carrier Service \\
\hline 1 & San Luis Obispo, Ca & East Lansing, MI & USPS Priority Mail \\
\hline 2 & San Luis Obispo, Ca & Clemson, SC & USPS Priority Mail \\
\hline 3 & San Luis Obispo, Ca & Gainesville, FL & USPS Priority Mail \\
\hline 4 & San Luis Obispo, Ca & Atlantic City, NJ & USPS Priority Mail \\
\hline 5 & San Luis Obispo, Ca & Washington DC & USPS Priority Mail \\
\hline 6 & San Luis Obispo, Ca & Berlin, Germany & DHL International Express \\
\hline 7 & San Luis Obispo, Ca & Rio De Janeiro, Brazil & DHL International Express \\
\hline 8 & San Luis Obispo, Ca & Melbourne, Australia & DHL International Express \\
\hline 9 & San Luis Obispo, Ca & Beijing, China & DHL International Express \\
\hline
\end{tabular}


After collecting the data of interest throughout each route of distribution, composite testing profiles representative of the complete distribution environment were developed. In essence, these newly developed testing profiles included data from all the different segments of transport used in the modern air parcel environment.

For climatic hazards, this included the identification of the experienced temperature, humidity, and atmospheric pressure levels in each route. From this data, profiles utilizing the level and duration of conditional extremes and averages could be developed.

For physical hazards, both the shock and vibration levels were analyzed. Shock was defined through the identification of the occurrence rate and the equivalent drop height that would need to occur to induce the same level of shock. It is determined through the use of the "zero g channel" and the "resultant velocity change" calculation methods. Further explanation of these calculation methodologies are discussed above (section 2.4.2).

For vibration, a single composite power spectral density (PDS) profile was created. This was accomplished by weighting the time the test packages spent within each mode of transport used throughout distribution. 


\subsection{RESULTS AND DISCUSSION}

At the completion of data collection, analysis was performed and composite testing profiles were created for each hazard element of vibration, shock, temperature, humidity, and atmospheric pressure. These newly developed testing profiles are representative of what is experienced throughout the entire air parcel distribution environment. They are built from hazard level data collected from every mode of transport encountered in a single distribution route. At a minimum, this included transport in vehicles such as delivery vans, tractor/trailer semi trucks, aircraft tarmac dollies, aircraft, and a number of the different types of material handling equipment used at hubs and sortation facilities. Detailed findings from the analysis of each of these hazards is expanded upon in the sections below.

\subsection{Climatic Hazard Findings}

For the climatic hazard levels of temperature, humidity, and atmospheric pressure, the data recorded at ten minute intervals was analyzed to identify the upper bounds, lower bounds, and the average levels of each condition encountered. This analysis identified temperature levels ranging from $8-45^{\circ} \mathrm{C}\left(46-113^{\circ} \mathrm{F}\right)$, humidity levels ranging from 21.9 to $92.7 \%$, and atmospheric pressure levels ranging from 102 to $78.6 \mathrm{kPa}$ (11.4 to 14.9 PSI). 
A complete summary of all the climatic conditions encountered in each route is presented in table 24 and 25.

Table 24: Temperature and humidity conditional averages

\begin{tabular}{|c|c|c|c|}
\hline \multicolumn{2}{|c|}{ Turnaround Destination } & \multirow{2}{*}{$\begin{array}{c}\begin{array}{c}\text { Average Temperature } \\
{ }^{\circ} \mathbf{C ~}\left({ }^{\circ} \mathrm{F}\right)\end{array} \\
23.1(73.6)\end{array}$} & \multirow{2}{*}{$\begin{array}{c}\begin{array}{c}\text { Average Humidity } \\
(\%)\end{array} \\
56.9\end{array}$} \\
\hline \multirow{6}{*}{$\begin{array}{l}\text { Domestic } \\
\text { Routes }\end{array}$} & Atlantic City, NJ & & \\
\hline & Clemson, SC & $23.3(56.9)$ & 56.9 \\
\hline & East Lansing, MI & $22.1(71.8)$ & 52.7 \\
\hline & Gainesville, FL & $23.5(74.3)$ & 53.6 \\
\hline & Washington D.C. & $22.7(72.9)$ & 57 \\
\hline & Average & 22.9 (73.3) & 55.4 \\
\hline \multirow{5}{*}{$\begin{array}{c}\text { International } \\
\text { Routes }\end{array}$} & Melbourne, Australia & $21.0(69.8)$ & 48.7 \\
\hline & Rio de Janeiro, Brazil & $23.9(75.0)$ & 61.2 \\
\hline & Beijing, China & $25.2(77.3)$ & 58.0 \\
\hline & Berlin, Germany & $23.9(75.1)$ & 55.4 \\
\hline & Average & $23.5(74.3)$ & 55.8 \\
\hline
\end{tabular}


Table 25: Minimum / maximum climatic condition levels

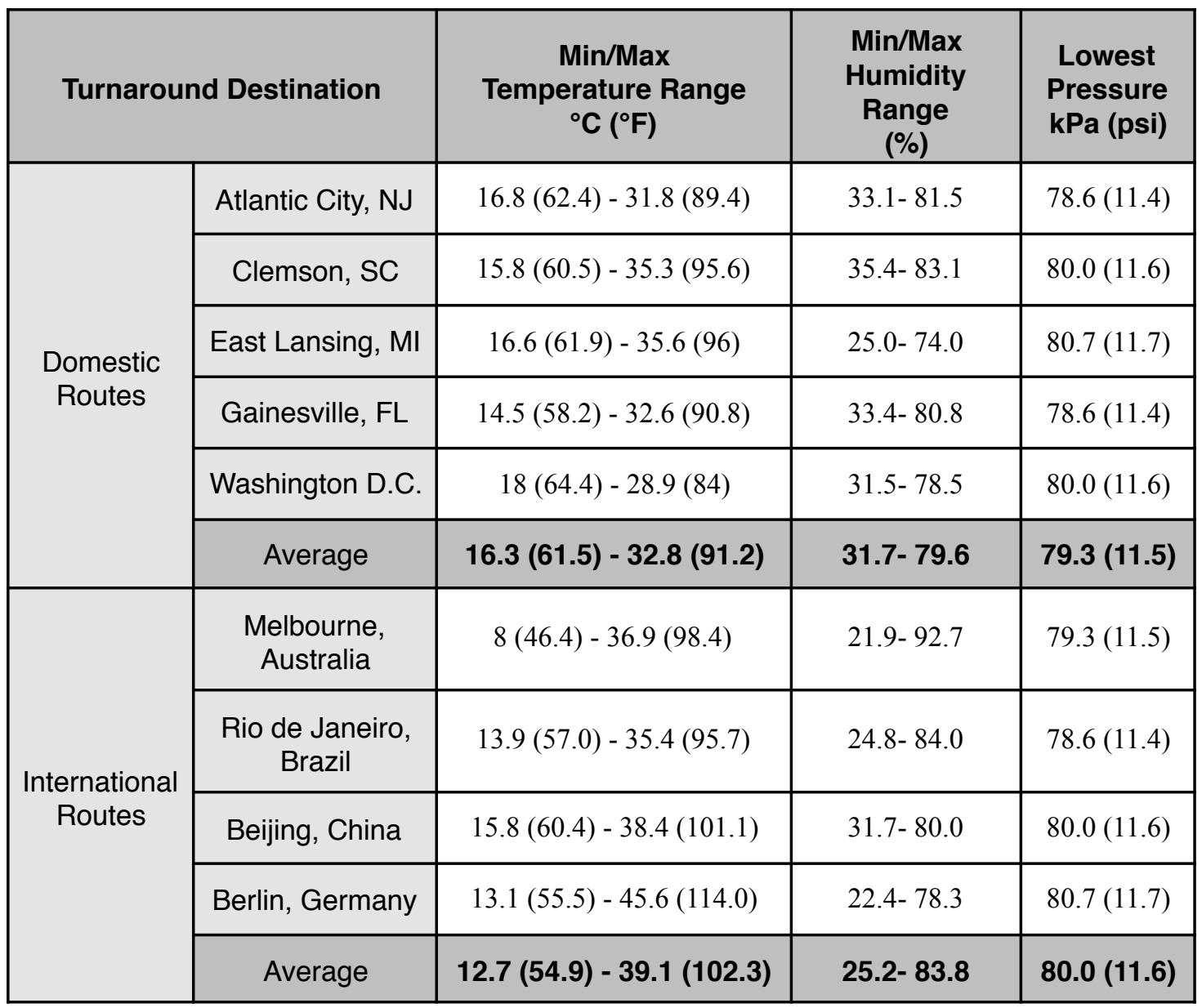

From this data, it can be identified that high altitude commercial jetliners were used for all segments of air transport. These types of aircraft have engineering controls built into the cargo holds that maintain consistent climatic conditions within them. This conclusion was developed after the identification of the average conditions experienced in both international and domestic routes to be fairly consistent with one another. The small deviations present can be attributed to ground transport through different 
geographic locations in vehicles that store packages in cargo holds that are not climatically controlled.

The finding of the lowest experienced pressure to be 11.4 PSI is especially suggestive of high altitude commercial jetliners being used for all segments of air transport. This is the pressure present at an altitude of only 1,950 m (6,400 ft) above sea level. In past studies, much lower pressure levels, indicative of higher altitudes, have been found within the feeder aircraft network. Although high altitude commercial jetliners operate at higher altitudes, the engineering control systems built into these aircraft maintain the climatic conditions experienced in them to a more moderate level. Although all the air transport exhibited trends of high altitude commercial jetliners, slight pressure variations were still found among the different routes of transport. These small variations are due to the performance level of the climatic control systems built into the different models of aircraft.

In addition to the lowest pressure findings, it is important to note all of the experienced pressure level change rates fell in accordance with the change rates outlined in the existing pressure testing standard- ASTM D6653-01 Standard Test Methods for Determining the Effects of High Altitude on Packaging Systems by Vacuum Method. This standard outlines pressure levels to change at a rate of $304.8 \mathrm{~m}$ (1000 ft.) every 30-60 
seconds. Within this study, all of the collected change rates fell in accordance with these values.

\subsubsection{Suggested Climatic Hazard Testing Updates}

Due to the findings described above, it is suggested to condition all test packages for a minimum of 24 hours in the conditions of $23^{\circ} \mathrm{C}$ and $50 \%$

$\mathrm{RH}$. If specific details of climatic hazard levels are known for any particular distribution route, the packages should be subjected to additional conditioning environments as outlined in ASTM D4332: Standard Practice for Conditioning Containers, Packages, or Packaging Components for Testing. Also, as no pressure levels of greater severity were found in this study, no changes are suggested to be made to the existing pressure testing standard, as outlined in ASTM D6653-01. In accordance with this standard, the pressure level of 7.25 PSI should be maintained for a minimum of 60 minutes.

\subsection{Physical Hazard Findings}

After collecting data throughout the various routes of distribution, the experienced vibration levels and incurred shock forces were quantified and analyzed. From this data, composite testing profiles representative of the hazard levels experienced throughout the entire air parcel distribution environment were created. For vibration, this included the development of a composite power spectral density (PDS) plot. For shock, both the amount of drops incurred, and the drop height occurrence level for $90 \%$, 
$95 \%$, and $99 \%$ of all the package drops were identified. From the analysis of this data, two drop testing sequences representing the levels of shock experienced throughout distribution were developed. Details of the findings from the analysis of these physical hazards is expanded upon in the sections below.

\subsubsection{Shock Findings}

For shock, a summary consisting of both the average total number of incurred impacts, as well as the occurrence rate of varying package drop heights is shown in table 26. This table includes the drop height occurrence level for $90 \%, 95 \%$, and $99 \%$ of all experienced drops. This occurrence level finding, can be read that $99 \%$ of the drops experienced were from below a height of $126 \mathrm{~cm}$ in domestic shipments and below a heigh of $154 \mathrm{~cm}$ in international shipments.

Table 26: Measured drop heights for all air shipment routes

\begin{tabular}{|c|c|c|}
\hline Drop Data & Domestic & International \\
\hline Average number of drops in a one-way trip & 15 & 13 \\
\hline Maximum drop height cm (in) & $215(84.8)$ & $193(75.9)$ \\
\hline Drop height at 99\% occurrence cm (in) & $126(49.8)$ & $154(60.6)$ \\
\hline Drop height at 95\% occurrence cm (in) & $72.6(28.6)$ & $89.4(35.2)$ \\
\hline Drop height at 90\% occurrence cm (in) & $56.6(22.3)$ & $51.6(20.3)$ \\
\hline Average drop height cm (in) & $24.1(9.5)$ & $23.6(9.3)$ \\
\hline
\end{tabular}

Although the majority of drops occurred from a height lower than this, five drops were observed to occur from a significantly greater height. The six highest drops experienced in the domestic and international routes are shown in descending 
order in table 27. As these peak heights ranged from 95.8 to $216 \mathrm{~cm}$, it can be concluded that drops of this height are not only uncommon, but also occur from a dramatically greater height than a majority of the drops experienced. For this reason, the developed drop testing sequence was based around the $99 \%$ occurrence level drop height of all the drops experienced. Through this analysis, these uncommon peak drop height events are considered outliers and are not recognized in this test specification as it has the objective of representing the "normal" air transport conditions.

Table 27: Highest measured drop heights for all air shipment routes

\begin{tabular}{|c|c|c|}
\hline Rank & $\begin{array}{c}\text { Domestic Drop Height } \\
\text { cm (in) }\end{array}$ & $\begin{array}{c}\text { International Drop Height } \\
\text { cm (in) }\end{array}$ \\
\hline Highest & $216(84.9)$ & $193(75.9)$ \\
\hline 2nd highest & $211(83.1)$ & $154(61.6)$ \\
\hline 3rd highest & $182(71.8)$ & $124(49.0)$ \\
\hline 4th highest & $126(49.8)$ & $118(46.3)$ \\
\hline 5th highest & $100(39.3)$ & $116(45.6)$ \\
\hline 6th highest & $95.8(37.7)$ & \\
\hline
\end{tabular}

Lastly, the package impact orientations were also analyzed. The proportions of the observed impact orientations is shown in table 28. From this information, it is apparent package edges incurred the greatest proportion of impact in domestic shipments and faces experienced the greatest proportion of impacts in 
international shipments. This finding leads towards the conclusion of a package in domestic transport receiving a greater amount of manual handling, and subsequently, a greater number of true vertical drops. Meanwhile, international shipments experienced a greater amount of handling through automated handling equipment and incurred a greater proportion of impacts on faces from impacts during horizontal movement when a packages slides and collides with the walls of handling machinery or other packages. Additionally, the conclusion made from the proportions of impact orientations in each shipment type is strengthened when comparing the drop heights incurred in both the domestic and international shipments. By looking at the data presented in table 27 above, it is apparent that packages transported through international routes were exposed to slightly less severe shock conditions than the packages traveling through domestic distribution. Not only did international routes have fewer total impacts, but they also had a lower overall average drop height.

Table 28: Proportion of impact orientations

\begin{tabular}{|c|c|c|}
\hline Drop Orientation & Domestic & International \\
\hline Face & 34.6 & 40.0 \\
\hline Edge & 46.7 & 36.9 \\
\hline Corner & 18.7 & 23.1 \\
\hline
\end{tabular}


After a review of the package drop data found in this study, the highest drop height experienced by $99 \%$ of the packages was $126 \mathrm{~cm}$ in domestic distribution and $154 \mathrm{~cm}$ in international distribution. In order to develop a test to simulate package drops, modifications were made to the existing drop test sequence as outlined in 49 CFR 178.603 - Drop test.

This newly developed drop test sequence can be found in table 29. It is recommended to subject new packaging components to this entire package drop test sequence.

Table 29: Proposed package drop test sequence

\begin{tabular}{|c|c|c|c|}
\hline \multirow{2}{*}{ Drop \# } & \multirow{2}{*}{ Drop Orientation } & \multicolumn{2}{|c|}{ Drop Height cm (in) } \\
\cline { 3 - 4 } & & Domestic & International \\
\hline $\mathbf{1}$ & Flat on bottom side & $127(50)$ & $155(61)$ \\
\hline $\mathbf{2}$ & Flat on top side & $127(50)$ & $155(61)$ \\
\hline $\mathbf{3}$ & Flat on long side & $127(50)$ & $155(61)$ \\
\hline $\mathbf{4}$ & Flat on short side & $127(50)$ & $155(61)$ \\
\hline $\mathbf{5}$ & On a corner & $127(50)$ & $155(61)$ \\
\hline
\end{tabular}

\subsubsection{Vibration Findings}

To quantify vibration, composite power density spectrum (PDS) profiles were developed. These newly developed profiles of random vibration represent the vibration levels experienced throughout the entire air parcel distribution environment.

To compile these vibration profiles, data was collected throughout all the segments of transport used in the modern air parcel distribution 
environment. By analyzing the package tracking information provided by the shipment carriers, the time proportions in table 30 were identified as the amount of time spent within each segment of transport. The identification of these time proportions allowed for development of a weighted average profile that represents a complete route of distribution.

Table 30: Allocation of time spent in various modes of transport

\begin{tabular}{|c|c|}
\hline Transport Mode & Time \\
\hline Pickup and Delivery Van & $1 \mathrm{~h}$ to $4 \mathrm{~h}$ \\
\hline Truck Shipment & $(0) 1 \mathrm{~h}$ to $6 \mathrm{~h}$ \\
\hline $\begin{array}{c}\text { Jet Aircraft } \\
\text { (Domestic Shipments) }\end{array}$ & $(0) 1 \mathrm{~h}$ to $5 \mathrm{~h}$ \\
\hline $\begin{array}{c}\text { Jet Aircraft } \\
\text { (International Shipments) }\end{array}$ & $1 \mathrm{~h}$ to $16 \mathrm{~h}$ \\
\hline Air Containers & 0 to $1 \mathrm{~h}$ \\
\hline
\end{tabular}

The breakpoints of these newly developed PDS curves can be found in table 31. Two sets of breakpoints are given for both international and domestic shipments that represent $1 \sigma$ (one sigma) and $3 \sigma$ (three sigma) of all the experienced vibration levels. 
Table 31: Breakpoints of developed PDS spectrums

\begin{tabular}{|c|c|c|c|c|}
\hline \multirow{2}{*}{ Frequency } & \multicolumn{2}{|c|}{$\begin{array}{c}\text { Domestic } \\
\text { Power Density }\left(g^{2} / \mathrm{Hz}\right)\end{array}$} & \multicolumn{2}{|c|}{$\begin{array}{c}\text { International } \\
\text { Power Density }\left(\mathrm{g}^{2} / \mathrm{Hz}\right)\end{array}$} \\
\hline & $\operatorname{PD}(\sigma)$ & PD (3б) & $\operatorname{PD}(\sigma)$ & PD (3б) \\
\hline 1 & 0.00005 & 0.00015 & 0.00005 & 0.00015 \\
\hline 2 & 0.0007 & 0.0021 & 0.0007 & 0.0021 \\
\hline 3 & 0.0033 & 0.0099 & 0.0033 & 0.0099 \\
\hline 4 & 0.01 & 0.03 & 0.01 & 0.03 \\
\hline 16 & 0.01 & 0.03 & 0.01 & 0.03 \\
\hline 25 & 0.003 & 0.009 & 0.003 & 0.009 \\
\hline 26 & 0.0419 & 0.1257 & 0.0254 & 0.0762 \\
\hline 60 & 0.0419 & 0.1257 & 0.0254 & 0.0762 \\
\hline 250 & 0.000124 & 0.000372 & 0.00008 & 0.00024 \\
\hline Average $G_{r m s}$ & 1.557 & 2.697 & 1.224 & 2.155 \\
\hline
\end{tabular}

Illustrations of these newly developed PDS profiles, overlaid with the profiles of existing standards, is given in Figures 17 and 18. Through the analysis of the illustration of these testing profiles, the frequencies with the most identifiable power density differential occurs between $25-60 \mathrm{hz}$. The greater acceleration levels present in these frequencies has not been captured in existing vibration test profiles. The absence of these levels is alarming as this acceleration could readily lead towards degradative damage in both the packaging and the products within. 


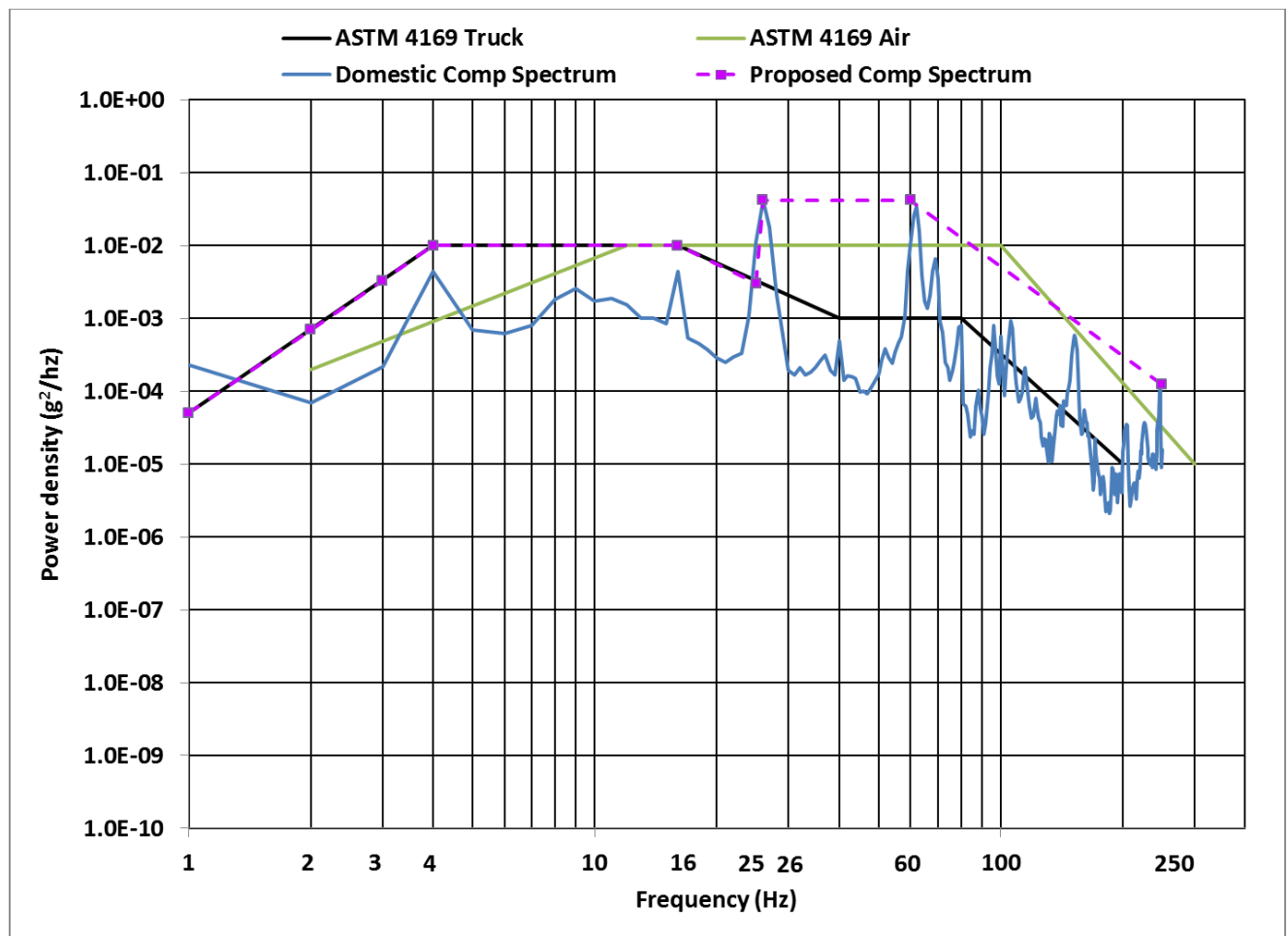

Figure 17: Composite PDS spectrum for domestic routes

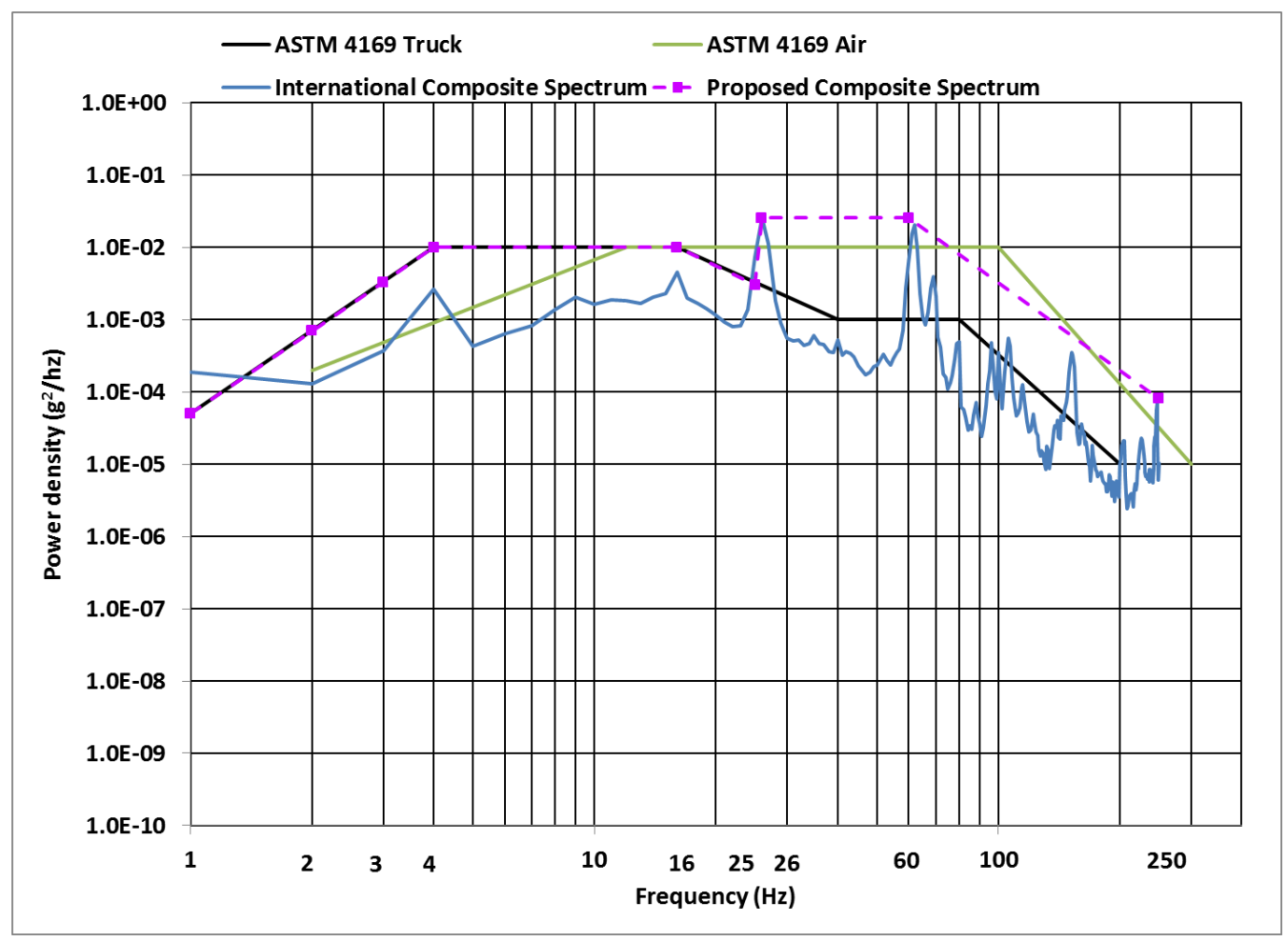

Figure 18: Composite PDS spectrum for international routes 
After analyzing the experienced vibration in each distribution route, a single power spectral density profile, representing the normal conditions encountered throughout the entire air parcel distribution environment, were developed. This random vibration PDS profile is to be used in accordance with ASTM D4728: Standard Test Method for Random Vibration Testing of Shipping Containers. The conditions defined in this profile represent $99.5 \%$ of the experienced vibration levels. As the vibration levels found in domestic and international routes were similar, this single profile is proposed for representation of both distribution types.

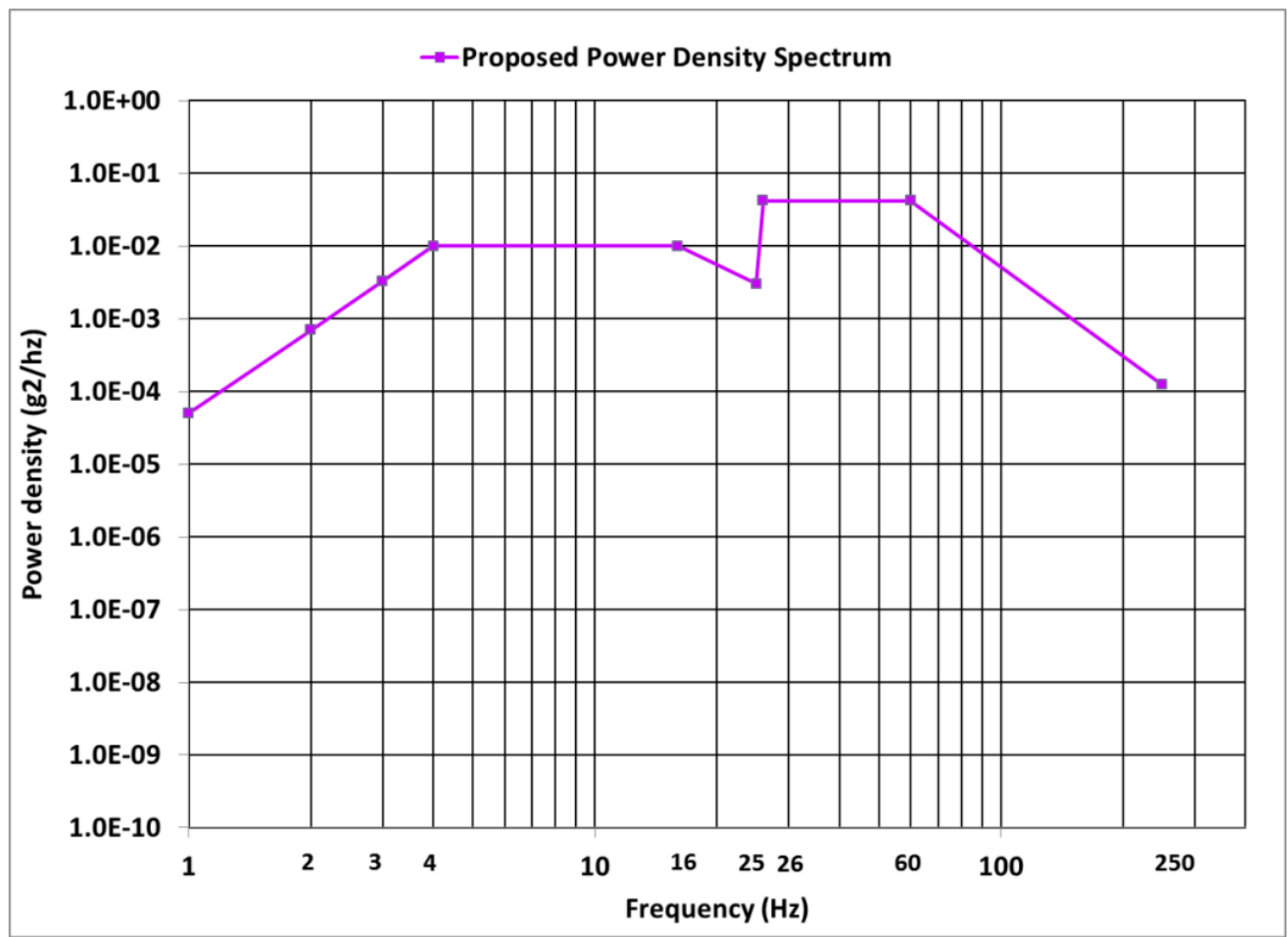

Figure 19: Proposed power density spectrum (PDS) test profile 
Testing with random vibration such as this is much more representative of actual distribution conditions when compared to the vibration testing standard currently outlined in the Code of Federal Regulations. Specifically, the vibration levels called for in the current test method, 49 CFR 178.608 - Vibration Standard, does not represent the type of vibration present in any mode of transport used in modern air parcel distribution. The vibration in this test method is a sinusoidal waveform conducted at a single frequency and at a consistent level of acceleration. Furthermore, the current test specifies to determine the appropriate test frequency through identifying the frequency that causes the test package to "bounce". This is a weak methodology to create a test to represent normal distribution as there is no evidence that a package is ever subjected to a repeated "bounce" in actual distribution. Instead, a random vibration profile, consisting of a multitude of frequencies at varying acceleration levels, is much more representative of the conditions actually experienced in normal distribution.

\subsection{Summary and Significance of Results}

At the conclusion of this study, testing profiles were developed to simulate the levels of temperature, humidity, atmospheric pressure, shock, and vibration experienced in "normal" modern air parcel distribution. In order to quantify these hazard levels, test packages were sent through distribution and data recorders monitored the hazard levels present.

Although package testing standards have been created and widely used in industry, the development of a single composite vibration profile that represents 
all modes of transport used throughout an entire route of distribution is an approach that has not been developed. In order to create this single vibration test profile, analysis was done on the vibration level experienced in each mode of transport and an accurate weighted average composite profile was developed based on the amount of time the package spends in each segment of transport. The creation of this testing profile allows for all sources of vibration to be properly accounted for during the development of new package designs.

\subsection{Suggested Further Research}

Throughout the completion of this study, several possible research extensions were identified. These modifications would allow the collected data to better represent the true hazard levels present. Some possible extensions include:

- The collection of data during all seasons in order to account for the effect of seasonal variability on both the physical and climatic hazard levels experienced in the "normal" air parcel distribution environment.

- In addition to gathering vibration data from data recorders mounted inside of test packages being sent through distribution, data recorders could also be mounted to the frame structure of the different transportation vehicles in order to collect direct input vibration levels present during vehicle movement.

- Although data was collected with multiple test packages being sent through each distribution route included in this study, collecting 
additional data from additional trips to additional destinations would further increase the representative strength of the collected data towards the true average hazard levels present.

- As it was observed all data collected in this study was from transport on high altitude commercial jetliners, the collection of additional hazard level data from low altitude feeder aircraft could be beneficial to help identify all the variations present in the complete modern air distribution environment. 


\section{REFERENCES}

[1] Singh, J., Singh, S. P., \& Saha, K. (2015). Evaluation of Vibration Profiles for ULD Dollies at Air-Cargo Sorting Hubs. Journal of Applied Packaging Research, 7(1). http://doi.org/10.14448/japr.03.0002

[2] Schueneman, H., Khangaldy, P., \& Schwinghammer, G. (2014, September). Package Integrity Testing: Common Tests and Applications. Retrieved from http://www.westpak.com/images/pdf/ Package\%20Integrity\%20Testing\%20Webinar_final_091814_.pdf

[3] World Air Cargo Forecast. (2014-2015). Retrieved from http:// www.boeing.com/resources/boeingdotcom/commercial/about-our-market/ cargo-market-detail-wacf/download-report/assets/pdfs/wacf.pdf

[4] Air Cargo. (2017).

Retrieved from http://www.iata.org/whatwedo/cargo/Pages/index.aspx

[5] ELECTRONIC CODE OF FEDERAL REGULATIONS. (2017, March 15). Retrieved from http://www.ecfr.gov/cgi-bin/text-idx? $\mathrm{SID}=112746 \mathrm{f0754856b772cede24d791e89d \& mc=true \& tpl=/ecfrbrowse/}$ Title49/49tab_02.tpl

[6] Silverman, K. (2012). Temperature mapping study of United States distribution systems. Rochester Institute of Technology. Retrieved from http:// scholarworks.rit.edu/cgi/viewcontent.cgi?article $=8357 \&$ context=theses

[7] Singh, S. P., Singh, J., Stallings, J., Burgess, G., \& Saha, K. (2009). Measurement and Analysis of Temperature and Pressure in High Altitude Air Shipments. PACKAGING TECHNOLOGY AND SCIENCE, (23), 35-46.

[8] UPS Professional Services. (1998). Temperature Information for use in the Small Parcel Environment. Retrieved from https://www.google.co $\mathrm{m} / \mathrm{url}$ ?sa $=\mathrm{t} \& \mathrm{rct}=\mathrm{j} \& \mathrm{q}=\&$ esrc $=\mathrm{s} \&$ source $=$ web $\& \mathrm{~cd}=1 \& \mathrm{ved}=0$ ahUKEwiyoJjLIOr MAhVP92M KHWxyBP0QFggdMAA\&url=https\%3A\%2F\%2Fcompass.ups. com\%2FWorkArea \%2FDownloadAsset.aspx\%3Fid\%3D931\&usg=AFQjC NG8NuXokixUiiHzlpEbOm 6CQghF3g

[9] Lampman, S. (2003). Characterization and Failure Analysis of Plastics. ASM International. Retrieved from https://books.google.com/books?id=RJWiilJ LdxYC\&dq=PLASTICS+SUSCEPTIBILITY+TEMPERATURE \&source=gb S_navlinks_S

[10] Paper: Moisture and Relative Humidity. (2005, May). Glatfelter. Retrieved from http://www.glatfelter.com/files/products/carbonless/ Moisture_Humidity.pdf

[11] Standard Practice for Conditioning Containers, Packages, or Packaging Components for Testing. (n.d.). ASTM International: Standards Worldwide, (D4332 - 14). 
[12] Altitude above Sea Level and Air Pressure. (n.d.). Retrieved from http:// www.engineeringtoolbox.com/air-altitude-pressure-d_462.html

[13] Standard Test Methods for Determining the Effects of High Altitude on Packaging Systems by Vacuum Method. (n.d.). ASTM International: Standards Worldwide, (D6653/D6653M - 13), 1-4.

[14] RANDOM VIBRATION-AN OVERVIEW. (n.d.). Retrieved from http:// www.emtengineering.com/wp-content/uploads/2013/04/Barry-ControlsRandom-Vibration.pdf

[15] Kipp, W. (2008, December). VIBRATION TESTING EQUIVALENCE. Retrieved from https://www.ista.org/forms/Vibration_Testing_EquivalenceKipp_2000.pdf

[16] THEORY: SINE SWEEP VS RANDOM VIBRATION TESTING. (n.d.). ISTA Certified Laboratory. Retrieved from http://www.sebert.nl/Brochure/ Theory_Sine_Sweep_VS_Random_vibration_testing.pdf

[17] Irvine, T. (2007, March 15). POWER SPECTRAL DENSITY UNITS: [G^2/ $\mathrm{Hz}$. Retrieved from http://vibrationdata.com/tutorials2/psd.pdf

[18] Jagit, S., S. Paul, S., \& Eric, J. (2006). Measurement and analysis of US truck vibration for leaf spring and air ride suspensions, and development of tests to simulate these conditions. Packaging Technology and Science, 19(6), 309-323. http://doi.org/10.1002/pts.732

[19] Vertical Vibration Test Systems. (n.d.). Retrieved from http:// www.lansmont.com/products/vibration/vertical/

[20] STALLINGS, J., SINGH, J., \& SINGH, S. P. (2010). Measurement and Analysis of Vehicle Vibration for Parcel Delivery Vehicles in Single Parcel Shipments. JOURNAL OF APPLIED PACKAGING RESEARCH, 3(2), 7582. Retrieved from http://docplayer.net/9538767-Journal-of-appliedpackaging-research.html

[21] Singh, S. P., Singh, J., Gaur, P., \& Saha, K. (2007). Measurement and Analysis of Vibration Levels on Warehouse and Retail Store Material Handling Equipment. Journal of Applied Packaging Research, 2(2), 103120. Retrieved from http://digitalcommons.calpoly.edu/cgi/viewcontent.cgi? article $=1003 \&$ context=it_fac

[22] Wallin, B. (2007), Developing a Random Vibration Profile Standard. Proceedings of 2007 IAPRI Symposium, Windsor, UK

[23] Dunno, K. (2008). ANALYSIS OF IN-FLIGHT VIBRATION FOR A TURBO PROPELLER AIRCAFT. CLEMSON UNIVERSITY TIGER PRINTS. Retrieved from http://tigerprints.clemson.edu/cgi/viewcontent.cgi? article $=1484 \&$ context $=$ all_theses

[24] Singh, S. P., Burgess, G. J., \& Singh, J. (2004). A new test method and pictorial markings for packages containing liquid dangerous goods in high 
altitude shipments. Journal of Testing and Evaluation, 32(5), 417-420. Retrieved from http://compass.astm.org.ezproxy.lib.calpoly.edu/download/ JTE12006.34413.pdf

[25] Kinematic Equations and Free Fall. (2016, July 7- 16). Retrieved from http:// www.physicsclassroom.com/class/1DKin/Lesson-6/Kinematic-Equationsand-Free-Fall

[26] Garcia-Romeu-Martinez, M.-A., Singh, S. P., Cloquell-Ballester, V.-A., \& Saha, K. (2007). Measurement and Analysis of International Air Parcel Shipping Environment for DHL and FedEx between Europe and United States, 20(6), 421-429. http://doi.org/10.1002/pts.775

[27] Garcia-Romeu-Martinez, M.-A., Singh, S. P., Cloquell-Ballester, V.-A., \& Saha, K. (2007). Measurement and Analysis of International Air Parcel Shipping Environment for DHL and FedEx between Europe and United States, 20(6), 421-429. http://doi.org/10.1002/pts.775

[28] Singh, S. P., Burgess, G., \& Singh, J. (2004). Measurement and analysis of the second-day air small and light-weight package shipping environment within Federal Express. Packaging Technology and Science, (17), 119127. http://doi.org/10.1002/pts.647

[29] Singh, S. P., \& Voss, T. (1992). Drop heights encountered in the Unite Parcel Service Small Parcel Environment in the United States. Journal of Testing and Evaluation, 20(5), 382-387. http://doi.org/10.1520/JTE11925J 